\title{
Hunting for heavy majorana neutrinos with lepton number violating signatures at LHC
}

\author{
Chao Guo, ${ }^{a}$ Shu-Yuan Guo, ${ }^{a}$ Zhi-Long Han, ${ }^{a}$ Bin $\mathrm{Li}^{a}$ and $\mathrm{Yi} \mathrm{Liao}^{a, b, c, d}$ \\ ${ }^{a}$ School of Physics, Nankai University, \\ Tianjin 3000\%1, China \\ ${ }^{b}$ CAS Key Laboratory of Theoretical Physics, Institute of Theoretical Physics, \\ Chinese Academy of Sciences, Beijing 100190, China \\ ${ }^{c}$ Synergetic Innovation Center for Quantum Effects and Applications, \\ Hunan Normal University, Changsha, Hunan 410081, China \\ ${ }^{d}$ Center for High Energy Physics, Peking University, \\ Beijing 100871, China \\ E-mail: chaog@mail.nankai.edu.cn, shyuanguo@mail.nankai.edu.cn, \\ hanzhilong@mail.nankai.edu.cn, libinae@mail.nankai.edu.com, \\ liaoy@nankai.edu.cn
}

ABSTRACT: The neutrinophilic two-Higgs-doublet model $(\nu 2 \mathrm{HDM})$ provides a natural way to generate tiny neutrino mass from interactions with the new doublet scalar $\Phi_{\nu}$ $\left(H^{ \pm}, H, A\right)$ and singlet neutrinos $N_{R}$ of $\mathrm{TeV}$ scale. In this paper, we perform detailed simulations for the lepton number violating (LNV) signatures at LHC arising from cascade decays of the new scalars and neutrinos with the mass order $m_{N_{R}}<m_{\Phi_{\nu}}$. Under constraints from lepton flavor violating processes and direct collider searches, their decay properties are explored and lead to three types of LNV signatures: $2 \ell^{ \pm} 4 j+\mathbb{E}_{T}, 3 \ell^{ \pm} 4 j+\mathbb{E}_{T}$, and $3 \ell^{ \pm} \ell^{\mp} 4 j$. We find that the same-sign trilepton signature $3 \ell^{ \pm} 4 j+\mathbb{E}_{T}$ is quite unique and is the most promising discovery channel at the high-luminosity LHC. Our analysis also yields the $95 \%$ C.L. exclusion limits in the plane of the $\Phi_{\nu}$ and $N_{R}$ masses at 13 (14) TeV LHC with an integrated luminosity of $100(3000) \mathrm{fb}^{-1}$.

Keywords: Beyond Standard Model, Neutrino Physics, Higgs Physics

ARXIV EPRINT: 1701.02463 


\section{Contents}

1 Introduction 1

2 Model and constraints 2

2.1 The model 2

2.2 Constraints 5

3 Decay properties $\quad 6$

3.1 Neutrinophilic scalars 8

3.2 Heavy Majorana neutrinos $\quad 9$

4 Lepton number violating signatures $\quad 12$

$\begin{array}{lll}4.1 & \text { Dilepton signature } & 14\end{array}$

$\begin{array}{lll}4.2 & \text { Trilepton signature } & 17\end{array}$

$\begin{array}{lll}4.3 & \text { Four-lepton signature } & 20\end{array}$

$\begin{array}{llr}5 & \text { Conclusion } & 21\end{array}$

\section{Introduction}

Neutrino mass and mixing provides robust evidence for physics beyond the standard model (SM). Regarding SM as a low energy effective field theory, the tiny neutrino mass can be incorporated by the unique dimension-five Weinberg operator [1]. The high energy scale realization of this operator remains however to be a theoretical puzzle. If it is realized at the tree level, there are three possibilities to do so [2], which correspond exactly to the canonical type I [3-7], type II [8-12], and type III seesaw [13] respectively. The new particles introduced in these seesaws are typically so heavy that they are even beyond the reach of high energy colliders such as the Large Hadron Collider (LHC). To achieve tiny neutrino mass at relatively lower scales, more sophisticated mechanisms have been proposed, such as inverse [14-16] and linear seesaws [17-19], pushing the effective neutrino mass operator to even higher dimensions [20-31], or attributing it to a purely radiative effect [32-111]; see, e.g., refs. [112, 113] for reviews. In these generalized mechanisms, new particles could be at a $\mathrm{TeV}$ scale, making their detection in principle possible at LHC.

The demand for very heavy Majorana neutrinos in the type I seesaw arises partly from the difficulty to arrange for a naturally small Yukawa coupling between the light and heavy neutrinos which yields a Dirac mass for neutrinos. To relax the tension, a second Higgs doublet $\Phi_{\nu}$ is introduced in the so-called neutrinophilic two-Higgs-doublet model $(\nu 2 \mathrm{HDM})$ [114-125]. By assigning a lepton number to $\Phi_{\nu}$ but not to the Majorana neutrinos $N_{R}$, the Yukawa coupling between them and the SM lepton doublet $L, \bar{L} \widetilde{\Phi}_{\nu} N_{R}$, is 
allowed by the lepton number $\mathrm{U}(1)_{L}$ symmetry, while the SM Higgs doublet $\Phi$ is forbidden to couple to $L$ and $N_{R}$. Assuming further $\mathrm{U}(1)_{L}$ is softly broken by a bilinear term $\Phi^{\dagger} \Phi_{\nu}$ in the scalar potential, $\Phi_{\nu}$ develops a naturally small vacuum expectation value (VEV) out of that of $\Phi$, so that a tiny neutrino mass becomes possible from a small Dirac mass without requiring a terribly heavy Majorana neutrino. Additional benefits such as a dark matter candidate [126-130] and leptogenesis [126, 131-135] have also been actively explored in the framework of $\nu 2 \mathrm{HDM}$.

The above discussion indicates that $\nu 2 \mathrm{HDM}$ can be considered as a type-I-like seesaw, but with relative larger Yukawa couplings [136] among the SM leptons and the new scalar and heavy Majorana neutrinos. The new particles can now be naturally at a TeV scale and could be within the reach of LHC. The usual lepton number violating (LNV) signature for heavy neutrinos at LHC, $p p \rightarrow \ell^{ \pm} N_{R} \rightarrow \ell^{ \pm} \ell^{ \pm} j j$, has been well studied [137-155], albeit with significant fine-tuning between tiny neutrino masses and detectable heavy-light neutrino mixing in type I seesaw (see, e.g., refs. [156, 157] for discussions on fine-tuning issues). In $\nu 2 \mathrm{HDM}$, the direct production of $\ell^{ \pm} N_{R}$ is still suppressed heavily by the small mixing, but now $N_{R}$ could also arise as a decay product of other new particles that can be copiously produced at LHC [158-172]. This is indeed the case when $N_{R}$ is lighter than $\Phi_{\nu}$ which can be pair or associated produced via the Drell-Yan process followed by its decay to $N_{R}$ via the Yukawa coupling. The further decay of $N_{R}, N_{R} \rightarrow \ell^{ \pm} W^{\mp}$, then triggers various LNV signatures at LHC [173]. The purpose of this work is to investigate these signatures by detailed simulations.

Our paper is organized as follows. In section 2, we introduce $\nu 2 \mathrm{HDM}$ and consider constraints from lepton flavor violation (LFV) and direct collider searches. Then in section 3, we study the decay properties of neutrinophilic scalars and heavy neutrinos. The detailed simulation of various LNV signatures at LHC is performed in section 4. Finally, our conclusions are presented in the last section 5 .

\section{$2 \quad$ Model and constraints}

\subsection{The model}

The $\nu 2 \mathrm{HDM}$ was previously suggested in ref. [114]. It introduces a new scalar doublet $\Phi_{\nu}$ that has the same quantum numbers as the SM Higgs doublet $\Phi$, and three right-handed singlet neutrinos $N_{R}$. A global $\mathrm{U}(1)_{L}$ symmetry is then imposed, under which $\Phi_{\nu}$ has lepton number $L=-1$ and $N_{R}$ has null lepton number. This then distinguishes between $\Phi_{\nu}$ and $\Phi$ : while $\Phi_{\nu}$ couples to $N_{R}, \Phi$ couples only to SM fermions, thus avoiding flavor changing neutral currents at tree level in the Yukawa sector. When a soft $\mathrm{U}(1)_{L}$ breaking term is introduced in the scalar potential, a small VEV of $\Phi_{\nu}$ can naturally develop, making $\nu 2 \mathrm{HDM}$ generically different from the conventional two-Higgs-doublet models [174].

Denoting the two scalar doublets as

$$
\Phi=\left(\begin{array}{c}
\phi^{+} \\
\left(v+\phi^{0, r}+i \phi^{0, i}\right) / \sqrt{2}
\end{array}\right), \Phi_{\nu}=\left(\begin{array}{c}
\phi_{\nu}^{+} \\
\left(v_{\nu}+\phi_{\nu}^{0, r}+i \phi_{\nu}^{0, i}\right) / \sqrt{2}
\end{array}\right)
$$


the scalar potential is

$$
\begin{aligned}
V= & -m_{\Phi}^{2} \Phi^{\dagger} \Phi+m_{\Phi_{\nu}}^{2} \Phi_{\nu}^{\dagger} \Phi_{\nu}+\frac{1}{2} \lambda_{1}\left(\Phi^{\dagger} \Phi\right)^{2}+\frac{1}{2} \lambda_{2}\left(\Phi_{\nu}^{\dagger} \Phi_{\nu}\right)^{2} \\
& +\lambda_{3}\left(\Phi^{\dagger} \Phi\right)\left(\Phi_{\nu}^{\dagger} \Phi_{\nu}\right)+\lambda_{4}\left(\Phi^{\dagger} \Phi_{\nu}\right)\left(\Phi_{\nu}^{\dagger} \Phi\right)-\left(\mu^{2} \Phi^{\dagger} \Phi_{\nu}+\text { H.c. }\right)
\end{aligned}
$$

Since vanishing of $\mu^{2}$ would enhance symmetry, it can be considered as naturally small. Assuming $m_{\Phi, \Phi_{\nu}}^{2}>0$ and $\lambda$ s fulfil various bounded-from-below conditions such as $\lambda_{1,2}>0$ and $\lambda_{1} \lambda_{2} \geq\left(\lambda_{3}+\lambda_{4}\right)^{2}[174,175], \Phi_{\nu}$ develops a small VEV out of that of $\Phi$ :

$$
v \simeq \sqrt{\frac{2 m_{\Phi}^{2}}{\lambda_{1}}}, v_{\nu} \simeq \frac{\mu^{2} v}{m_{\Phi_{\nu}}^{2}+\left(\lambda_{3}+\lambda_{4}\right) v^{2} / 2} .
$$

To get some feel about the seesaw-like relation between the VEVs $v_{\nu}$ and $v$, we may assume, for instance, $m_{\Phi_{\nu}} \sim 500 \mathrm{GeV}, \mu^{2} \sim 10 \mathrm{GeV}^{2}$ to arrive at $v_{\nu} \sim 10 \mathrm{MeV}$. Since $\mu^{2}$ is the only source of $\mathrm{U}(1)_{L}$ breaking, its radiative correction is proportional to itself and only logarithmically sensitive to the cutoff [118]. The hierarchy $v_{\nu} \ll v$ is thus stable against radiative corrections $[132,176,177]$.

After electroweak symmetry breaking, the physical scalars are given by

$$
\begin{aligned}
H^{ \pm} & =\phi^{ \pm} \sin \beta-\phi_{\nu}^{ \pm} \cos \beta, & A & =\phi^{0, i} \sin \beta-\phi_{\nu}^{0, i} \cos \beta, \\
H & =\phi^{0, r} \sin \alpha-\phi_{\nu}^{0, r} \cos \alpha, & h & =-\phi^{0, r} \cos \alpha-\phi_{\nu}^{0, r} \sin \alpha,
\end{aligned}
$$

where the mixing angles $\beta$ and $\alpha$ are determined by

$$
\tan \beta=\frac{v_{\nu}}{v}, \tan 2 \alpha \simeq 2 \frac{v_{\nu}}{v} \frac{-\mu^{2}+\left(\lambda_{3}+\lambda_{4}\right) v v_{\nu}}{-\mu^{2}+\lambda_{1} v v_{\nu}},
$$

and their masses are

$$
m_{H^{ \pm}}^{2} \simeq m_{\Phi_{\nu}}^{2}+\frac{1}{2} \lambda_{3} v^{2}, m_{A}^{2} \simeq m_{H}^{2} \simeq m_{H^{ \pm}}^{2}+\frac{1}{2} \lambda_{4} v^{2}, m_{h}^{2} \simeq 2 m_{\Phi}^{2}=\lambda_{1} v^{2} .
$$

Since $v_{\nu} \ll v$ in our consideration here, $\Phi$ is almost identical with the SM Higgs doublet, and $h$ can be regarded as the boson of mass $m_{h}=125 \mathrm{GeV}$ discovered at LHC [178-180]. For simplicity, we will assume in our numerical analysis a degenerate mass spectrum of $\Phi_{\nu}$, i.e., $m_{H^{ \pm}}=m_{H}=m_{A}=m_{\Phi_{\nu}}$, which can be realized by taking $\lambda_{3}=\lambda_{4}=0$. This simplification trivially saturates the bounded-from-below conditions mentioned above.

Under the $\mathrm{U}(1)_{L}$ symmetry, the Yukawa coupling and the Majorana mass terms for $N_{R}$ are given by

$$
-\mathcal{L}_{N}=y \bar{L} \widetilde{\Phi}_{\nu} N_{R}+\frac{1}{2} \overline{N_{R}^{c}} m_{N_{R}} N_{R}+\text { H.c. }
$$

with $\widetilde{\Phi}_{\nu}=i \sigma_{2} \Phi_{\nu}^{*}$. The coupling $y$ will be fixed by phenomenologically known neutrino parameters and theoretically expected values for $v_{\nu}$ and $m_{N_{R}}$. Without loss of generality, we assume the charged leptons and $N_{R}$ have been diagonalized. Note that the same Yukawa coupling can also be obtained by imposing a discrete $\mathbb{Z}_{2}$ symmetry [121]. But the $\mathbb{Z}_{2}$ scenario is found to be in severe tension with the electroweak precision tests, while the 
$\mathrm{U}(1)_{L}$ scenario is still viable [181]. Analogously to the type I seesaw, the mass matrix for light neutrinos can be derived approximately from eq. (2.8):

$$
m_{\nu}=-\frac{1}{2} v_{\nu}^{2} y m_{N_{R}}^{-1} y^{T}=U_{\mathrm{PMNS}} \hat{m}_{\nu} U_{\mathrm{PMNS}}^{T}
$$

where $\hat{m}_{\nu}=\operatorname{diag}\left(m_{1}, m_{2}, m_{3}\right)$ is the diagonalized neutrino mass matrix and $U_{\text {PMNS }}$ is the usual Pontecorvo-Maki-Nakagawa-Sakata (PMNS) matrix:

$$
\begin{aligned}
U_{\mathrm{PMNS}}= & \left(\begin{array}{ccc}
c_{12} c_{13} & s_{12} c_{13} & s_{13} e^{i \delta} \\
-s_{12} c_{23}-c_{12} s_{23} s_{13} e^{-i \delta} & c_{12} c_{23}-s_{12} s_{23} s_{13} e^{-i \delta} & s_{23} c_{13} \\
s_{12} s_{23}-c_{12} c_{23} s_{13} e^{-i \delta} & -c_{12} s_{23}-s_{12} c_{23} s_{13} e^{-i \delta} & c_{23} c_{13}
\end{array}\right) \\
& \times \operatorname{diag}\left(e^{i \varphi_{1} / 2}, 1, e^{i \varphi_{2} / 2}\right) .
\end{aligned}
$$

Here the shortcuts $c_{i j}=\cos \theta_{i j}$ and $s_{i j}=\sin \theta_{i j}$ are used, $\delta$ is the Dirac phase and $\varphi_{1,2}$ are the two Majorana phases. In our numerical sampling we will consider neutrino masses in either normal $(\mathrm{NH})$ or inverted hierarchy $(\mathrm{IH})$, and scan randomly the oscillation parameters in the $2 \sigma$ ranges of table I in ref. [182].

The Yukawa coupling matrix $y$ in eq. (2.9) can be solved in terms of neutrino parameters, $v_{\nu}, m_{N_{R}}$, and free parameters in the Casas-Ibarra parametrization [183, 184]:

$$
y=\frac{\sqrt{2}}{v_{\nu}} U_{\mathrm{PMNS}} \hat{m}_{\nu}^{1 / 2} R m_{N_{R}}^{1 / 2}
$$

where $R$ is a generalized orthogonal matrix:

$$
R=\left(\begin{array}{ccc}
u_{21} & -\omega_{21} & 0 \\
\omega_{21} & u_{21} & 0 \\
0 & 0 & 1
\end{array}\right)\left(\begin{array}{ccc}
u_{31} & 0 & -\omega_{31} \\
0 & 1 & 0 \\
\omega_{31} & 0 & u_{31}
\end{array}\right)\left(\begin{array}{ccc}
1 & 0 & 0 \\
0 & u_{32} & -\omega_{32} \\
0 & \omega_{32} & u_{32}
\end{array}\right)
$$

with $u_{i j}=\left(1-\omega_{i j}^{2}\right)^{1 / 2}$ and $-1 \leq \omega_{i j} \leq 1$ when $R$ is real. Then the mixing matrix between heavy and light neutrinos can be expressed as [185]

$$
V_{\ell N}=\frac{y v_{\nu}}{\sqrt{2}} m_{N_{R}}^{-1}=U_{\mathrm{PMNS}} \hat{m}_{\nu}^{1 / 2} R m_{N_{R}}^{-1 / 2}
$$

Differently from the type I seesaw, the small neutrino mass may be attributed to a small $v_{\nu}$ instead of a large $m_{N_{R}}$ or a tiny $y$, making heavy Majorana neutrinos possibly within the reach of LHC. For instance, $m_{\nu} \sim 0.01 \mathrm{eV}$ can be obtained with $v_{\nu} \sim 10 \mathrm{MeV}$, $m_{N_{R}} \sim 200 \mathrm{GeV}$, and $y \sim 0.006$ whence we have $V_{\ell N} \sim 10^{-7}$. As will be clear in section 2.2, the choice of $v_{\nu} \sim 10 \mathrm{MeV}$ mainly arises from tight constraints on lepton flavor violation. For such a small $v_{\nu}$, the modification to the SM Higgs properties due to the mixing between the doublet scalars is far below the current LHC sensitivity [186] and can be safely ignored. With our simplifying assumption of $\lambda_{3,4} \approx 0$, the charged scalars $H^{ \pm}$do not contribute to the diphoton decay $h \rightarrow \gamma \gamma$ either. In a word, no anomalous SM Higgs decays would be observable in this scenario. 

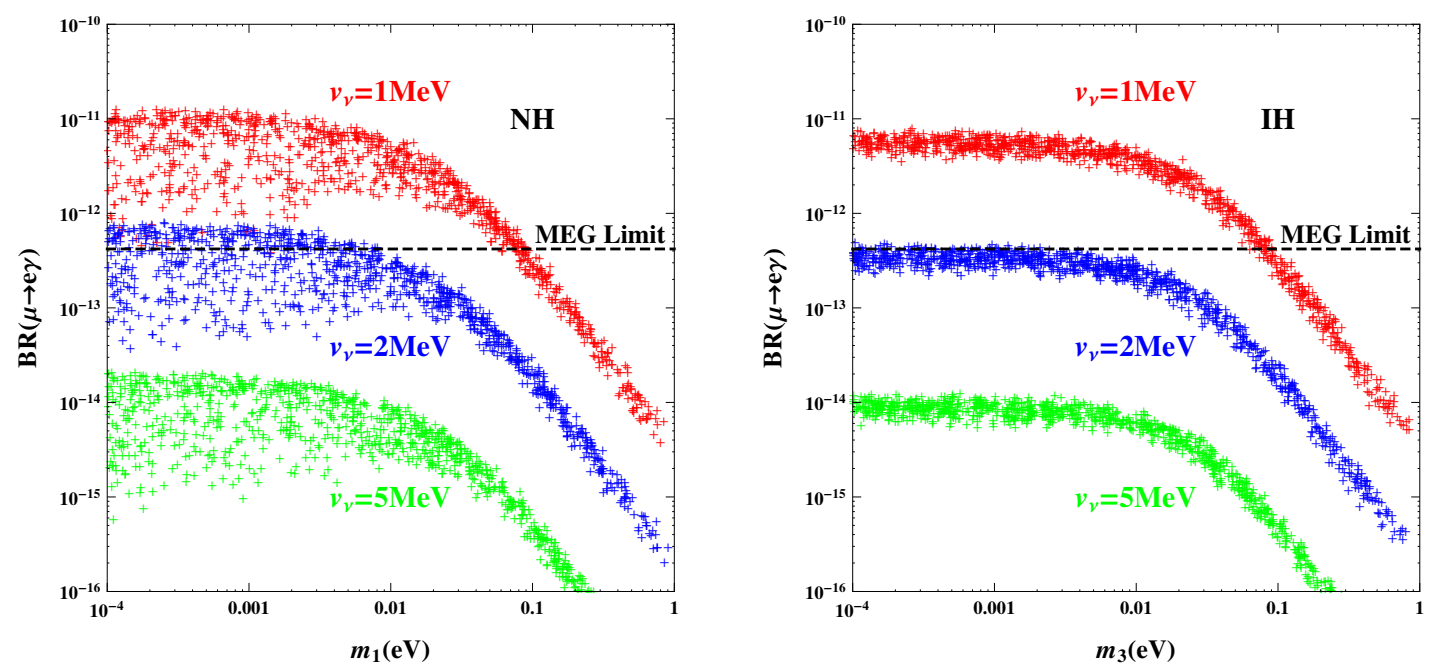

Figure 1. $\operatorname{BR}(\mu \rightarrow e \gamma)$ as a function of the lightest neutrino mass for normal (left panel) and inverted (right) hierarchy at $\left(m_{H^{+}}, m_{N_{R}}\right)=(300,200) \mathrm{GeV}$.

\subsection{Constraints}

Now we consider experimental constraints on the $\nu 2 \mathrm{HDM}$. Cosmological considerations have been used to set an upper bound on the sum of neutrino masses, $\sum_{i} m_{i}<0.23 \mathrm{eV}[187$, 188]. The null result searching for neutrinoless double- $\beta$ decays requires the standard effective neutrino mass $\langle m\rangle_{e e}=\left|\sum_{i}\left(V_{\mathrm{PMNS}}\right)_{e i}^{2} m_{i}\right|$ to be less than $0.061-0.165 \mathrm{eV}[189-$ 194], while the additional contribution from $\Phi_{\nu}$ is negligible due to its neutrinophilic nature. It is thus safe to state that the lightest neutrino mass should be less than $0.1 \mathrm{eV}$.

The Yukawa coupling $y \bar{L} \widetilde{\Phi}_{\nu} N_{R}$ with a not too small $y$ can mediate measurable LFV processes $[195,196]$. The currently most stringent constraint comes from the MEG experiment on the decay $\mu \rightarrow e \gamma$ with $\operatorname{BR}(\mu \rightarrow e \gamma)<4.2 \times 10^{-13}[197,198]$, which is calculated as $[195,199]$

$$
\operatorname{BR}(\mu \rightarrow e \gamma)=\frac{3 \alpha}{64 \pi G_{F}^{2}}\left|\sum_{i} \frac{y_{\mu i} y_{e i}^{*}}{m_{H^{+}}^{2}} F\left(\Delta_{H^{+}}^{N_{R i}}\right)\right|^{2},
$$

where $\Delta_{B}^{A}=m_{A}^{2} / m_{B}^{2}$ is the ratio of the squared masses and the loop function $F(x)$ is

$$
F(x)=\frac{1}{6(1-x)^{4}}\left(1-6 x+3 x^{2}+2 x^{3}-6 x^{2} \ln x\right) .
$$

While nondegenerate $N_{R}$ s are required to generate nondegenerate light neutrinos, we assume approximately in our numerical estimates that they are nearly degenerate to reduce the number of free parameters. The branching ratio then simplifies to

$$
\operatorname{BR}(\mu \rightarrow e \gamma) \approx \frac{3 \alpha}{16 \pi G_{F}^{2}} \frac{m_{N_{R}}^{2}\left|\widetilde{m}_{\mu e}\right|^{2}}{m_{H^{+}}^{4} v_{\nu}^{4}}\left|F\left(\Delta_{H^{+}}^{N_{R}}\right)\right|^{2}
$$

with $\widetilde{m}=U_{\mathrm{PMNS}} \hat{m}_{\nu} U_{\mathrm{PMNS}}^{\dagger}$ and in particular

$$
\tilde{m}_{\mu e}=c_{12} c_{13} c_{23} s_{12}\left(m_{2}-m_{1}\right)+c_{13} s_{13} s_{23} e^{-i \delta}\left[\left(m_{3}-m_{2}\right)+c_{12}^{2}\left(m_{2}-m_{1}\right)\right] .
$$


Note that the dependence on the free Majorana phases and real matrix $R$ drops out in this approximation, but the dependence on the Dirac phase $\delta$ can be significant for LFV [200] as the mixing angle $\theta_{13}$ is known to be not small.

We show in figure $1 \mathrm{BR}(\mu \rightarrow e \gamma)$ as a function of the lightest neutrino mass in either $\mathrm{NH}$ or $\mathrm{IH}$ and for $\left(m_{H^{+}}, m_{N_{R}}\right)=(300,200) \mathrm{GeV}$. A too small value of $v_{\nu} \sim 1 \mathrm{MeV}$ is obviously in conflict with the MEG bound. For nearly degenerate light neutrinos with the lightest mass $m_{1 / 3} \gtrsim 0.01 \mathrm{eV}$, we have $m_{j}-m_{i} \approx \Delta m_{j i}^{2} /\left(2 m_{i}\right)$, which explains why $\left|\tilde{m}_{\mu e}\right|$ and thus $\operatorname{BR}(\mu \rightarrow e \gamma)$ decrease as $m_{1 / 3}$ increases. On the other hand, for the lightest mass $m_{1 / 3} \lesssim 0.01 \mathrm{eV}$, we have $\left|m_{j}-m_{i}\right| \approx \sqrt{\left|\Delta m_{j i}^{2}\right|}$ with $m_{i}$ being the lightest mass, so that $\widetilde{m}_{\mu e}$ and thus $\operatorname{BR}(\mu \rightarrow e \gamma)$ saturate to a constant. Using eq. (2.16), the upper bound on $\mathrm{BR}(\mu \rightarrow e \gamma)$ can be translated into a lower bound on $m_{H^{+}} v_{\nu}$ for a given $m_{N_{R}}$ :

$$
m_{H^{+}} v_{\nu} \gtrsim\left[\frac{1 \times 10^{-13}}{\operatorname{BR}(\mu \rightarrow e \gamma)}\left(\frac{m_{N_{R}}}{100 \mathrm{GeV}}\right)^{2}\right]^{1 / 4} \times 600 \mathrm{GeV} \cdot \mathrm{MeV}
$$

with $F\left(m_{N_{R}}^{2} / m_{H^{+}}^{2}\right) \sim 0.1$ when $m_{N_{R}} \sim m_{\Phi_{\nu}}$. Hence, for $m_{N_{R}} \sim 200 \mathrm{GeV}$ for instance, the current MEG limit implies that $m_{H^{+}} v_{\nu} \gtrsim 600 \mathrm{GeV} \cdot \mathrm{MeV}$, which can also be seen in figure 1. Note that due to the existence of heavy Majorana neutrino $N_{R}$, the lower bound on $v_{\nu}$ is about $\sqrt{m_{N_{R}} / m_{\nu}} \sim 10^{6}$ times higher than those on the VEV of the Higgs triplet in type II seesaw [201-203] or of the neutrinophilic doublet in the Dirac scenario of $\nu 2 \mathrm{HDM}[196]$.

Next we summarize direct collider searches for the new scalar doublet $\Phi_{\nu}$ and heavy Majorana neutrinos $N_{R}$. The collider signatures of $\nu 2 \mathrm{HDM}$ have been studied in refs. [118, $121,173,204]$, which concentrated mainly on the charged scalars $H^{ \pm}$. When $m_{N_{R}}>m_{H^{+}}$, the dominant decay of $H^{+}$would be $H^{+} \rightarrow \ell^{+} \nu$. The direct searches for signatures such as $\ell^{+} \ell^{-}+\mathbb{E}_{T}$ at LHC have excluded the region of $m_{H^{+}} \lesssim 300 \mathrm{GeV}[205,206]$. In the opposite case of $m_{N_{R}}<m_{H^{+}}$, the dominant decay of $H^{+}$would be $H^{+} \rightarrow \ell^{+} N_{R}$ with $N_{R}$ further decaying into $\ell^{ \pm} W^{\mp}, \nu Z$, and $\nu h$. A brief discussion on this case has been carried out in refs. [121, 173], however no searches for such signatures have been performed by experiments. Here we consider the loose LEP bound $m_{H^{+}}>80 \mathrm{GeV}$ [207]. Since heavy Majorana neutrinos $N_{R}$ also exist in the type I seesaw, their searches have been extensively studied via the LNV signature $p p \rightarrow \ell^{ \pm} N_{R} \rightarrow \ell^{ \pm} \ell^{ \pm} j j$ [140-142, 145-147, 151, 152, 158]. For $m_{N_{R}}<m_{W}$, LEP excluded $\left|V_{\ell N}\right|^{2} \gtrsim 2 \times 10^{-5}$ [208, 209], while for heavier $N_{R}$, LHC could give the most restrictive direct limits, i.e., $\left|V_{\ell N}\right|^{2}<0.017$ at $m_{N_{R}}=200 \mathrm{GeV}[210-$ 212]. In $\nu 2 \mathrm{HDM}$, the mixing $V_{\ell N}$ is predicted as $V_{\ell N}=U_{\mathrm{PMNS}} \hat{m}_{\nu}^{1 / 2} R m_{N_{R}}^{-1 / 2} \sim 10^{-7}$ for electroweak scale $m_{N_{R}}$ without fine-tuning, which is far below the current limits.

\section{Decay properties}

The decay properties of the neutrinophilic scalars $H^{ \pm}, H, A$ and heavy Majorana neutrinos $N_{R}$ have been discussed in refs. [114, 121]. For completeness, we first review briefly the scenario with $m_{N_{R}}>m_{\Phi_{\nu}}$. Then we concentrate on the opposite case with $m_{N_{R}}<m_{\Phi_{\nu}}$, where LNV signatures at LHC can arise. 

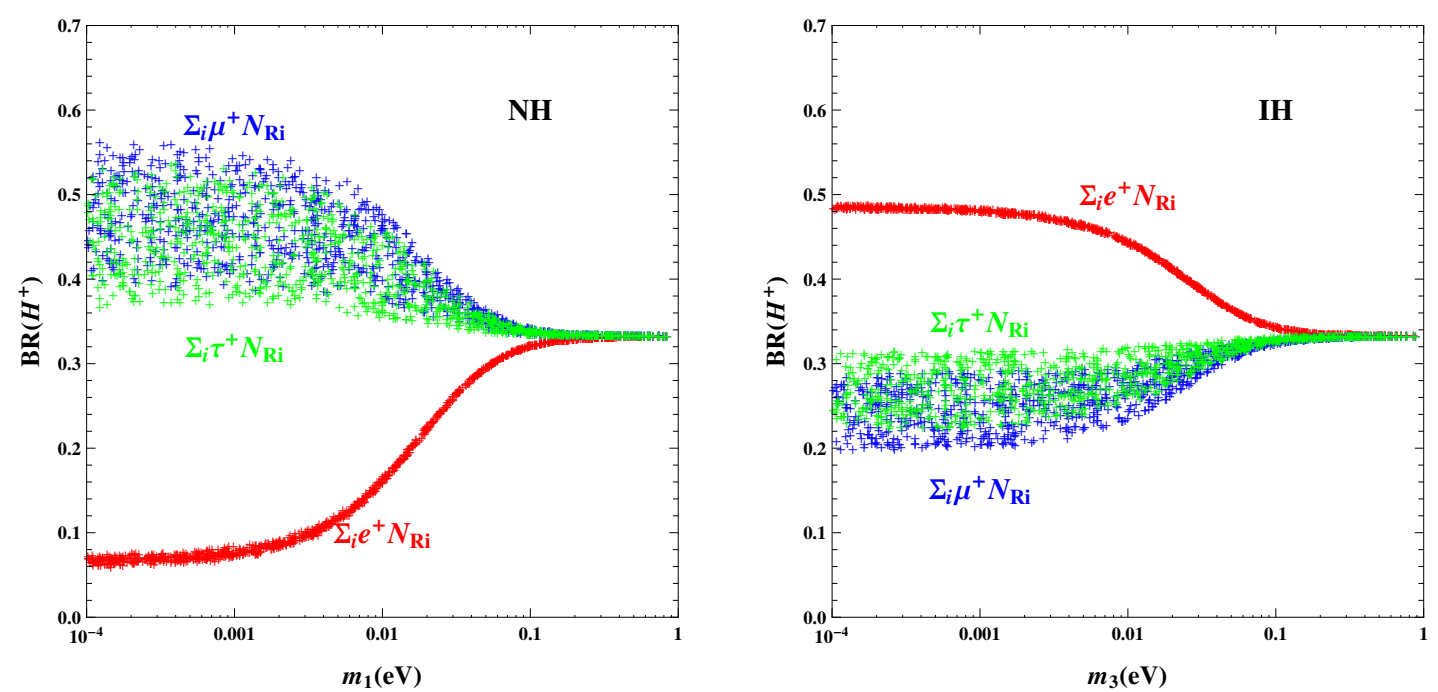

Figure 2. Branching ratios of the charged scalar $H^{+}$as a function of the lightest neutrino mass for normal (left panel) and inverted (right) hierarchy.

\begin{tabular}{|c|c|c|c|c|c|c|c|c|c|}
\hline $\mathrm{BR}\left(H^{+}\right)$ & $e^{+} N_{R 1}$ & $e^{+} N_{R 2}$ & $e^{+} N_{R 3}$ & $\mu^{+} N_{R 1}$ & $\mu^{+} N_{R 2}$ & $\mu^{+} N_{R 3}$ & $\tau^{+} N_{R 1}$ & $\tau^{+} N_{R 2}$ & $\tau^{+} N_{R 3}$ \\
\hline $\mathrm{NH}$ & 0.023 & 0.004 & 0.050 & 0.163 & 0.211 & 0.139 & 0.060 & 0.006 & 0.343 \\
\hline $\mathrm{IH}$ & 0.358 & 0.015 & 0.109 & 0.005 & 0.192 & 0.027 & 0.009 & 0.197 & 0.088 \\
\hline
\end{tabular}

Table 1. Branching ratios of the charged scalar $H^{+}$into $\ell^{+} N_{R i}$ for $\mathrm{NH}$ and $\mathrm{IH}$ at $m_{1 / 3}=0.001 \mathrm{eV}$ and $\omega_{i j}=0.5$.

When $m_{N_{R}}>m_{\Phi_{\nu}}, N_{R}$ decays dominantly into $\ell^{ \pm} H^{\mp}$ and $\nu H / A$. The singlet nature of $N_{R}$ makes it hardly producible at LHC and thus practically undetectable, but the doublet scalars can be pair or associated produced via the Drell-Yan processes. The scalars may decay as $H^{+} \rightarrow \ell^{+} \nu$ and $H / A \rightarrow \nu \nu$ via the small mixing $V_{\ell N}$ between the heavy and light neutrinos, which would lead to the same dilepton signature $\ell^{+} \ell^{-}+\mathbb{E}_{T}$ as in the Dirac type $\nu 2 \mathrm{HDM}[118,204]$. But this is not the case for the Majorana type $\nu 2 \mathrm{HDM}$ with $\mathrm{U}(1)_{L}$ symmetry when we take into account the constraints from LFV. From section 2.2, we know that $v_{\nu} \gtrsim 1 \mathrm{MeV}$ should be satisfied for $m_{\Phi_{\nu}}$ around the electroweak scale, and then the neutrinophilic scalars decay dominantly as $H^{+} \rightarrow c \bar{b} / t \bar{b} / W^{+} h, H \rightarrow b \bar{b} / t \bar{t} / h h$, and $A \rightarrow b \bar{b} / t \bar{t} / Z h$ when $v / v_{\nu} \lesssim 10^{5}$ [121]. The neutrinophilic scalars in this case are therefore long-lived since both $V_{\ell N}$ and $v_{\nu}$ are tiny, and the decays of $H^{ \pm}$could lead to detectable displaced vertices at LHC.

Now we turn to the more interesting scenario $m_{N_{R}}<m_{\Phi_{\nu}}$. In this scenario, we can safely neglect the mixing between $\Phi_{\nu}$ and $\Phi$ with $v_{\nu} \sim \mathcal{O}(10 \mathrm{MeV})[121]$, so that the dominant decays of the neutrinophilic scalars are $H^{+} \rightarrow \ell^{+} N_{R}$ and $H / A \rightarrow \nu N_{R}$, with heavy Majorana neutrinos $N_{R}$ decaying further into $\ell^{ \pm} W^{\mp}, \nu Z$, and $\nu h$. These decays are analyzed in the following subsections. 


\begin{tabular}{|c|c|c|c|}
\hline $\mathrm{BR}(H / A)$ & $\nu N_{R 1}$ & $\nu N_{R 2}$ & $\nu N_{R 3}$ \\
\hline $\mathrm{NH}$ & 0.246 & 0.221 & 0.533 \\
\hline $\mathrm{IH}$ & 0.372 & 0.404 & 0.224 \\
\hline
\end{tabular}

Table 2. Branching ratios of the neutral scalars $H / A$ into $\nu N_{R i}$ for NH and IH at $m_{1 / 3}=0.001 \mathrm{eV}$, $\omega_{i j}=0.5$ and upon summing over neutrinos $\nu$.

\subsection{Neutrinophilic scalars}

In the scenario of $m_{N_{R}}<m_{\Phi_{\nu}}$, the neutrinophilic scalars decay into charged leptons or neutrinos and heavy Majorana neutrino $N_{R}$ via the Yukawa coupling $y$. The partial decay widths are

$$
\begin{aligned}
\Gamma\left(H^{+} \rightarrow \ell^{+} N_{R i}\right) & =\frac{\left|y_{\ell i}\right|^{2}}{16 \pi} m_{H^{+}}\left(1-\Delta_{H^{+}}^{N_{R i}}\right)^{2}, \\
\Gamma\left(H / A \rightarrow \nu_{\ell} N_{R i}\right) & =\frac{\left|y_{\ell i}\right|^{2}}{16 \pi} m_{H / A}\left(1-\Delta_{H / A}^{N_{R i}}\right)^{2} .
\end{aligned}
$$

The branching ratios of the neutrinophilic scalars are only proportional to $\left|y_{\ell i}\right|^{2}$ and are then determined by the neutrino parameters via eq. (2.11). As mentioned earlier, we randomly scan the oscillation parameters in their $2 \sigma$ ranges of ref. [182] when evaluating the branching ratios. In figure 2 , we show the scanning results of $\operatorname{BR}\left(H^{+} \rightarrow \ell^{+} N_{R i}\right)$ as a function of the lightest neutrino mass $m_{1 / 3}$ in normal/inverted hierarchy by summing over the heavy Majorana neutrinos. We learn that in the nondegenerate neutrino mass region $m_{1 / 3} \lesssim 0.1 \mathrm{eV}$ :

$$
\begin{aligned}
& \sum_{i} \mathrm{BR}\left(H^{+} \rightarrow e^{+} N_{R i}\right)<\sum_{i} \mathrm{BR}\left(H^{+} \rightarrow \mu^{+} N_{R i}\right) \approx \sum_{i} \mathrm{BR}\left(H^{+} \rightarrow \tau^{+} N_{R i}\right) \text { for } \mathrm{NH} \\
& \sum_{i} \mathrm{BR}\left(H^{+} \rightarrow e^{+} N_{R i}\right)>\sum_{i} \mathrm{BR}\left(H^{+} \rightarrow \mu^{+} N_{R i}\right) \approx \sum_{i} \mathrm{BR}\left(H^{+} \rightarrow \tau^{+} N_{R i}\right) \text { for } \mathrm{IH}
\end{aligned}
$$

So we expect that the neutrino mass hierarchy might be distinguishable at LHC by the decay products of the charged scalars $H^{ \pm}$. To illustrate this, we use the best-fit values of the neutrino oscillation parameters in ref. [182] with the lightest neutrino mass $m_{1 / 3}=$ $0.001 \mathrm{eV}$ and $\omega_{i j}=0.5$ for the orthogonal $R$ matrix to evaluate $\operatorname{BR}\left(H^{+} \rightarrow \ell^{+} N_{R i}\right)$. The results are shown in table 1 for both $\mathrm{NH}$ and $\mathrm{IH}$, and will be employed for later signature simulations. From the table we are informed that a large hierarchy of individual branching ratios exists for both hierarchies with the largest being $\mathrm{BR}\left(H^{+} \rightarrow \tau^{+} N_{R 3}\right)=0.343$ for $\mathrm{NH}$ and $\operatorname{BR}\left(H^{+} \rightarrow e^{+} N_{R 1}\right)=0.358$ for $\mathrm{IH}$.

Concerning the neutral scalars $H$ and $A$, we sum over the light neutrinos when showing the scanning results, since they are invisible at colliders. In figure $3, \mathrm{BR}\left(H / A \rightarrow \nu N_{R i}\right)$ is shown as a function of the lightest neutrino mass for $\mathrm{NH}$ and $\mathrm{IH}$. No specific hierarchy in branching ratios is observed. A small difference between $\mathrm{NH}$ and $\mathrm{IH}$ is that the individual $\mathrm{BR}\left(H / A \rightarrow \nu N_{R i}\right)$ can exceed 0.5 in $\mathrm{NH}$ while it maximally reaches $0.5 \mathrm{in} \mathrm{IH.} \mathrm{The} \mathrm{explicit}$ values of $\operatorname{BR}\left(H / A \rightarrow \nu N_{R i}\right)$ are shown in table 2 using the same set of parameters as for $\operatorname{BR}\left(H^{+} \rightarrow \ell^{+} N_{R i}\right)$. It is clear from the table that the largest individual branching 

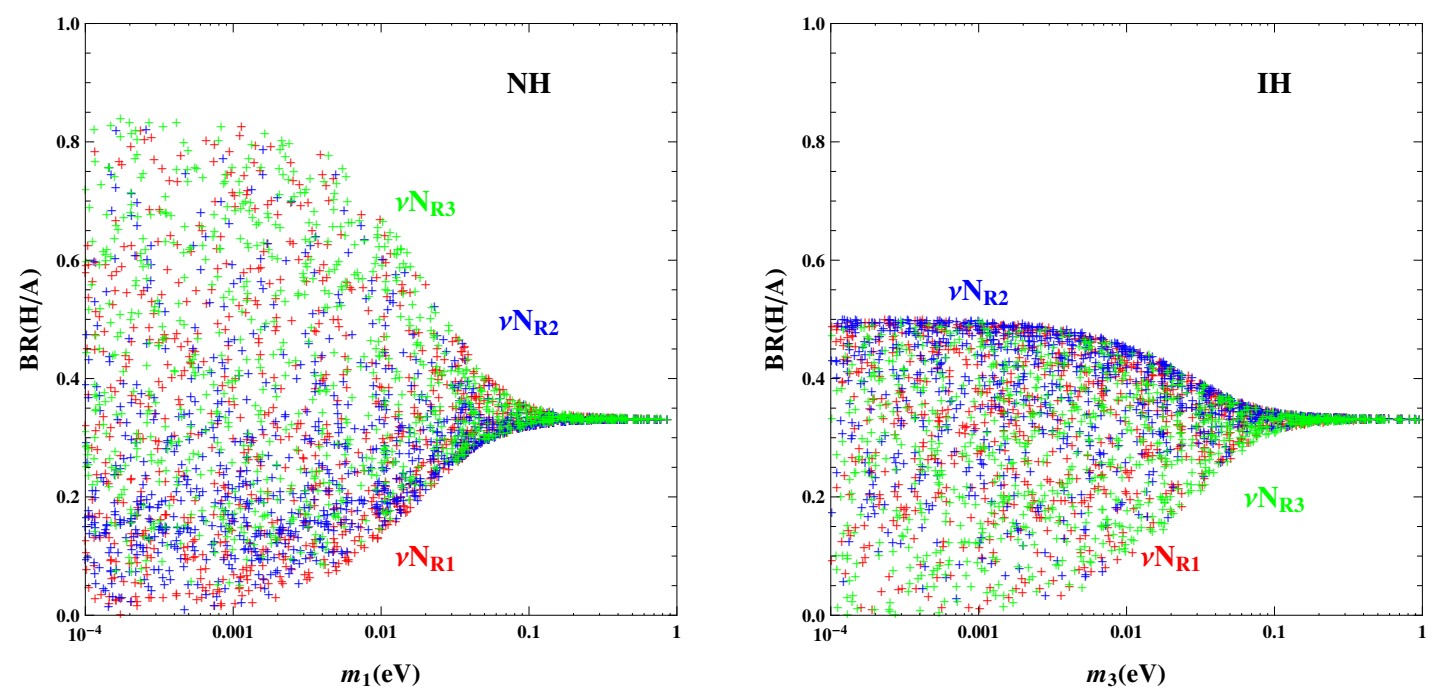

Figure 3. Branching ratios of the neutral scalars $H / A$ as a function of the lightest neutrino mass for normal (left panel) and inverted (right) hierarchy.

ratio of $H / A$ is $\mathrm{BR}\left(H / A \rightarrow \nu N_{R 3}\right)=0.533$ for $\mathrm{NH}$ and $\mathrm{BR}\left(H / A \rightarrow \nu N_{R 2}\right)=0.404$ for IH. And all branching ratios of $H / A \rightarrow \nu N_{R i}$ approach $1 / 3$ when the light neutrinos are nearly degenerate.

\subsection{Heavy Majorana neutrinos}

When the heavy Majorana neutrinos are lighter than the neutrinophilic scalars, they decay via the small mixing with the light neutrinos. The partial decay widths are given by

$$
\begin{aligned}
\Gamma\left(N_{R i} \rightarrow \ell^{ \pm} W^{\mp}\right) & =\frac{\left|V_{\ell i}\right|^{2}}{8 \pi v^{2}} m_{N_{R i}}^{3}\left(1-\Delta_{N_{R i}}^{W}\right)^{2}\left(1+2 \Delta_{N_{R i}}^{W}\right), \\
\Gamma\left(N_{R i} \rightarrow \nu_{\ell} Z\right) & =\frac{\left|V_{\ell i}\right|^{2}}{16 \pi v^{2}} m_{N_{R i}}^{3}\left(1-\Delta_{N_{R i}}^{Z}\right)^{2}\left(1+2 \Delta_{N_{R i}}^{Z}\right), \\
\Gamma\left(N_{R i} \rightarrow \nu_{\ell} h\right) & =\frac{\left|V_{\ell i}\right|^{2}}{16 \pi v^{2}} m_{N_{R i}}^{3}\left(1-\Delta_{N_{R i}}^{h}\right)^{2} .
\end{aligned}
$$

Due to the smallness of $V_{\ell N}, N_{R i}$ are actually long-lived, which could lead to visible displaced vertices at LHC [185]. In collider phenomenology study, the decay $N_{R i} \rightarrow \ell^{ \pm} W^{\mp}$ receives more attention, not only because it induces LNV signatures but also because it can be used to fully reconstruct the mass of $N_{R i}$. In figure 4, we present the branching ratios of $N_{R i}$ as a function of $m_{N_{R i}}$ upon summing over the lepton flavors, which depend only on heavy neutrino masses $m_{N_{R i}}$. As shown clearly, we have

$$
\operatorname{BR}\left(N_{R i} \rightarrow \ell^{ \pm} W^{\mp}\right): \operatorname{BR}\left(N_{R i} \rightarrow \nu Z\right): \mathrm{BR}\left(N_{R i} \rightarrow \nu h\right) \approx 2: 1: 1,
$$

in the large $m_{N_{R i}}$ limit, where the gauge bosons in the final state are mainly longitudinally polarized [142].

For the decays into charged leptons there are similar flavor relations among $\operatorname{BR}\left(N_{R i} \rightarrow\right.$ $\left.\ell^{ \pm} W^{\mp}\right)$ as in the decays of $H^{+}$. In figure 5 , the scanning results of $\operatorname{BR}\left(N_{R 1} \rightarrow \ell^{ \pm} W^{\mp}\right)$ 


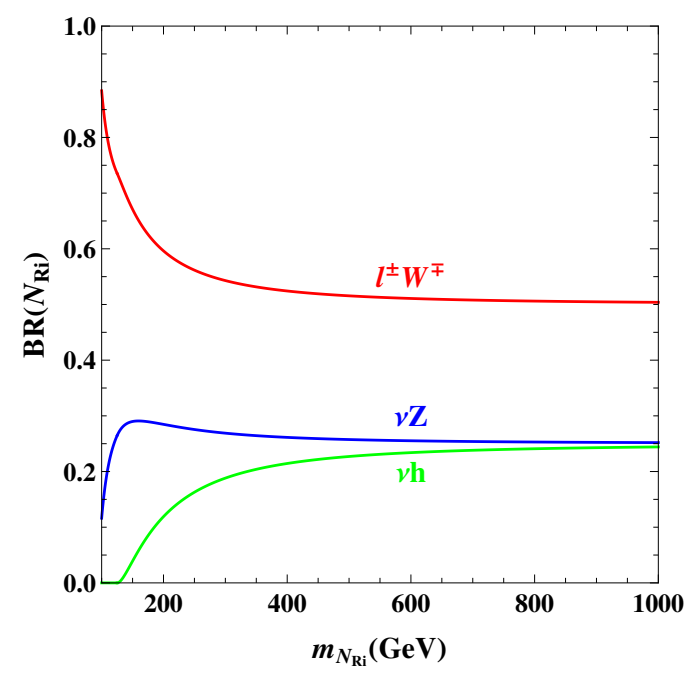

Figure 4. Branching ratios of $N_{R i}$ as a function of $m_{N_{R i}}$ upon summing over the lepton flavors.
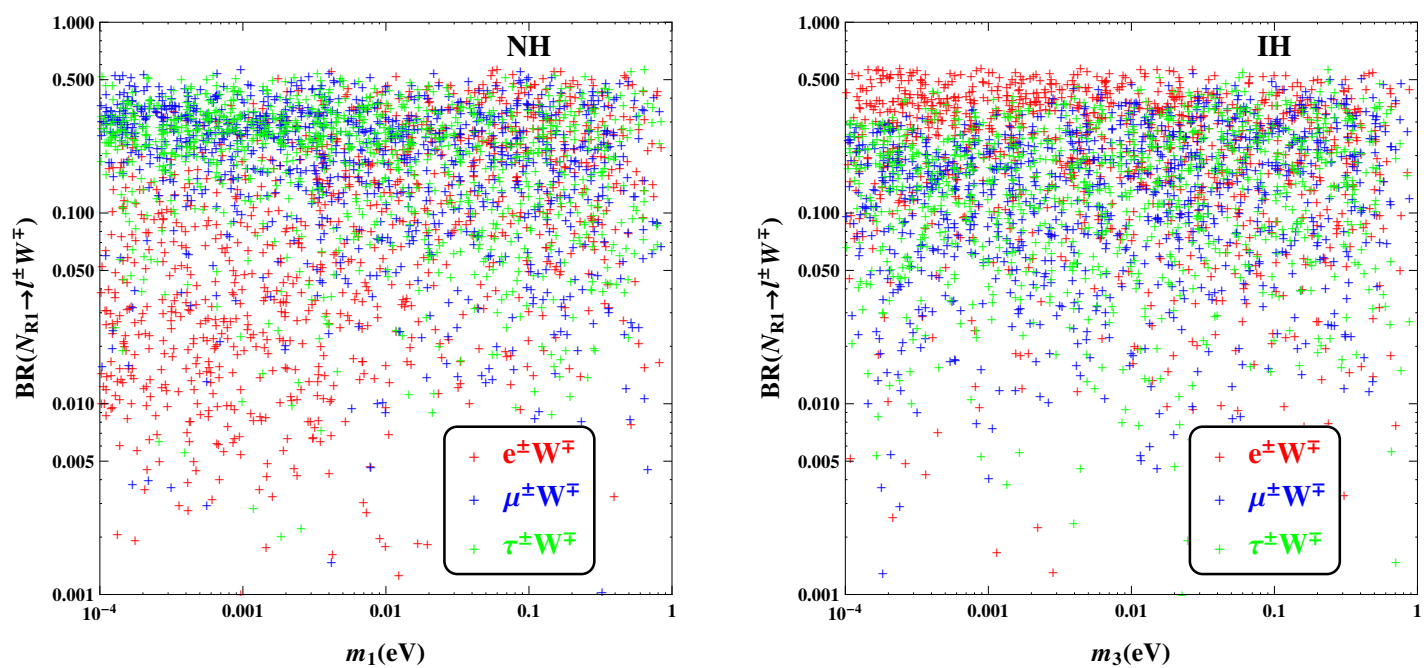

Figure 5. Branching ratios of $N_{R 1}$ into $\ell^{ \pm} W^{\mp}$ as a function of the lightest neutrino mass for normal (left panel) and inverted (right) hierarchy at $m_{N_{R 1}}=200 \mathrm{GeV}$.

are shown for $\ell=e, \mu, \tau$ separately as a function of the lightest neutrino mass at $m_{N_{R 1}}=$ $200 \mathrm{GeV}$, while the decays of $N_{R 2}$ and $N_{R 3}$ are similar. We observe the relations

$$
\begin{aligned}
& \mathrm{BR}\left(N_{R 1} \rightarrow e^{ \pm} W^{\mp}\right)<\mathrm{BR}\left(N_{R 1} \rightarrow \mu^{ \pm} W^{\mp}\right) \approx \mathrm{BR}\left(N_{R 1} \rightarrow \tau^{ \pm} W^{\mp}\right) \text { for } \mathrm{NH}, \\
& \operatorname{BR}\left(N_{R 1} \rightarrow e^{ \pm} W^{\mp}\right)>\operatorname{BR}\left(N_{R 1} \rightarrow \mu^{ \pm} W^{\mp}\right) \approx \mathrm{BR}\left(N_{R 1} \rightarrow \tau^{ \pm} W^{\mp}\right) \text { for } \mathrm{IH},
\end{aligned}
$$

when the lightest neutrino mass is less than $0.1 \mathrm{eV}$. These flavor relations can be better seen in figure 6 which shows the results of the averaged branching ratios $\sum_{i} \mathrm{BR}\left(N_{R i} \rightarrow\right.$ $\left.\ell^{ \pm} W^{\mp}\right) / 3$. Hence the decays of heavy Majorana neutrinos $N_{R i}$ into charged leptons could also be employed to distinguish between the neutrino mass hierarchies. Since $\operatorname{BR}\left(N_{R i} \rightarrow\right.$ 

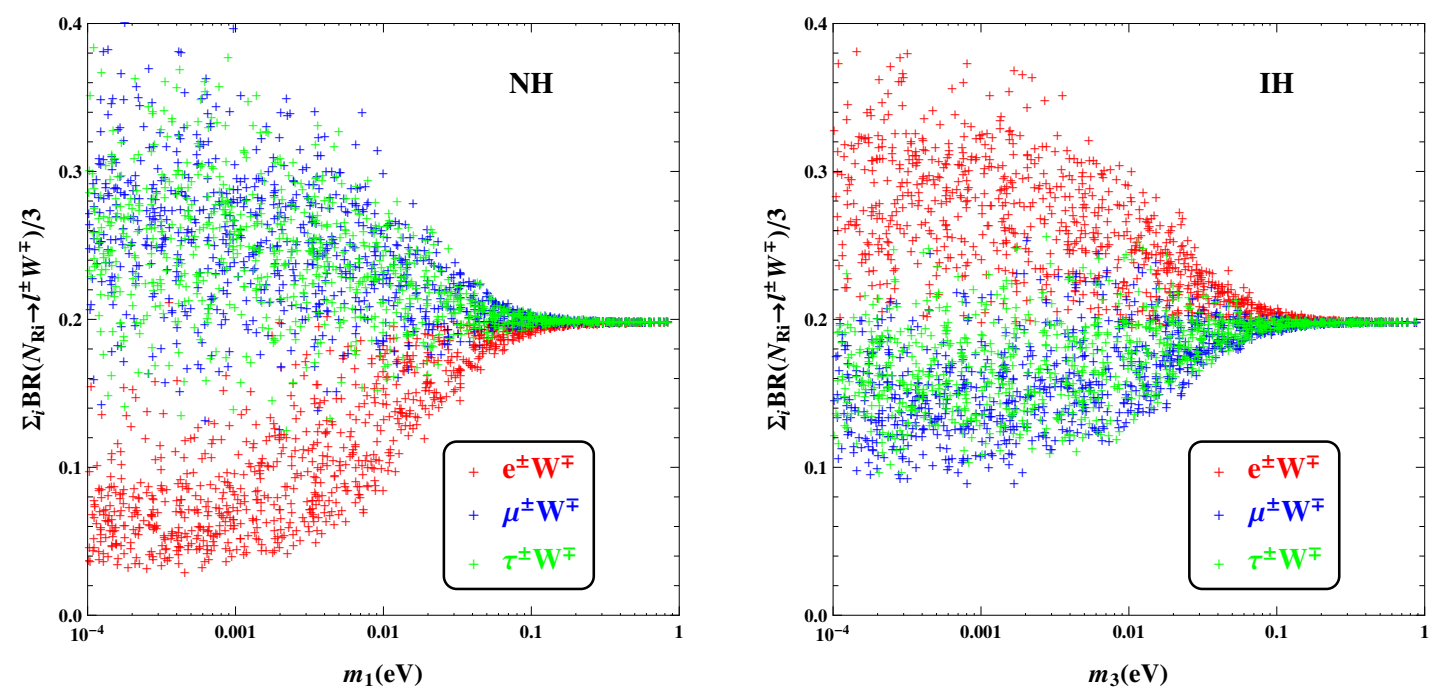

Figure 6. The averaged branching ratios of $N_{R i}$ as a function of the lightest neutrino mass for normal (left panel) and inverted hierarchy (right) at $m_{N_{R i}}=200 \mathrm{GeV}$.

\begin{tabular}{|c|c|c|c|}
\hline $\mathrm{FR}\left(N_{R i}\right)$ & $e^{ \pm} W^{\mp}$ & $\mu^{ \pm} W^{\mp}$ & $\tau^{ \pm} W^{\mp}$ \\
\hline$N_{R 1}$ & $0.093(0.962)$ & $0.663(0.014)$ & $0.244(0.024)$ \\
\hline$N_{R 2}$ & $0.019(0.037)$ & $0.955(0.476)$ & $0.026(0.487)$ \\
\hline$N_{R 3}$ & $0.094(0.486)$ & $0.261(0.122)$ & $0.645(0.392)$ \\
\hline
\end{tabular}

Table 3. Flavor ratios for heavy Majorana neutrinos $N_{R i}$ decaying into $\ell^{ \pm} W^{\mp}$ for $\mathrm{NH}(\mathrm{IH})$.

$\left.\ell^{ \pm} W^{\mp}\right)$ depends on $m_{N_{R i}}$, we define the flavor ratio (FR)

$$
\operatorname{FR}\left(N_{R i} \rightarrow \ell^{ \pm} W^{\mp}\right)=\frac{\operatorname{BR}\left(N_{R i} \rightarrow \ell^{ \pm} W^{\mp}\right)}{\sum_{\ell} \operatorname{BR}\left(N_{R i} \rightarrow \ell^{ \pm} W^{\mp}\right)}=\frac{\left|V_{\ell i}\right|^{2}}{\sum_{\ell}\left|V_{\ell i}\right|^{2}},
$$

which is independent of $m_{N_{R i}}$ but depends only on the neutrino oscillation parameters and the $R$ matrix. The distributions of $\operatorname{FR}\left(N_{R i} \rightarrow \ell^{ \pm} W^{\mp}\right)$ and $\sum_{i} \operatorname{FR}\left(N_{R i} \rightarrow \ell^{ \pm} W^{\mp}\right) / 3$ are similar to figures 5 and 6 respectively, up to a normalization factor of $\sum_{\ell} \mathrm{BR}\left(N_{R i} \rightarrow\right.$ $\left.\ell^{ \pm} W^{\mp}\right)$. In table 3 , we show the values of $\mathrm{FR}\left(N_{R i} \rightarrow \ell^{ \pm} W^{\mp}\right)$ for the same set of parameters as for $\operatorname{BR}\left(H^{+} \rightarrow \ell^{+} N_{R i}\right)$. For $\mathrm{NH}, N_{R 1,2} \rightarrow \mu^{ \pm} W^{\mp}$ and $N_{R 3} \rightarrow \tau^{ \pm} W^{\mp}$ are dominant while for IH $N_{R 1} \rightarrow e^{ \pm} W^{\mp}, N_{R 2} \rightarrow \mu^{ \pm} W^{\mp} / \tau^{ \pm} W^{\mp}$, and $N_{R 3} \rightarrow e^{ \pm} W^{\mp} / \tau^{ \pm} W^{\mp}$ take over. With the flavor ratios introduced above, we can easily acquire the branching ratios of $N_{R i} \rightarrow \ell^{ \pm} W^{\mp}$ for any values of $m_{N_{R i}}$ by

$$
\operatorname{BR}\left(N_{R i} \rightarrow \ell^{ \pm} W^{\mp}\right)=\operatorname{FR}\left(N_{R i} \rightarrow \ell^{ \pm} W^{\mp}\right) \times \sum_{\ell} \operatorname{BR}\left(N_{R i} \rightarrow \ell^{ \pm} W^{\mp}\right),
$$

where $\operatorname{FR}\left(N_{R i} \rightarrow \ell^{ \pm} W^{\mp}\right)$ and $\sum_{\ell} \mathrm{BR}\left(N_{R i} \rightarrow \ell^{ \pm} W^{\mp}\right)$ are given in table 3 and figure 4 , respectively.

Last but not least, we show in figure 7 the scatter plots for $\operatorname{BR}\left(H^{+} \rightarrow \ell^{+} \ell^{+} W^{-}\right)$ upon summing over the intermediate heavy Majorana neutrinos $N_{R i}$. With $W^{-}$further 

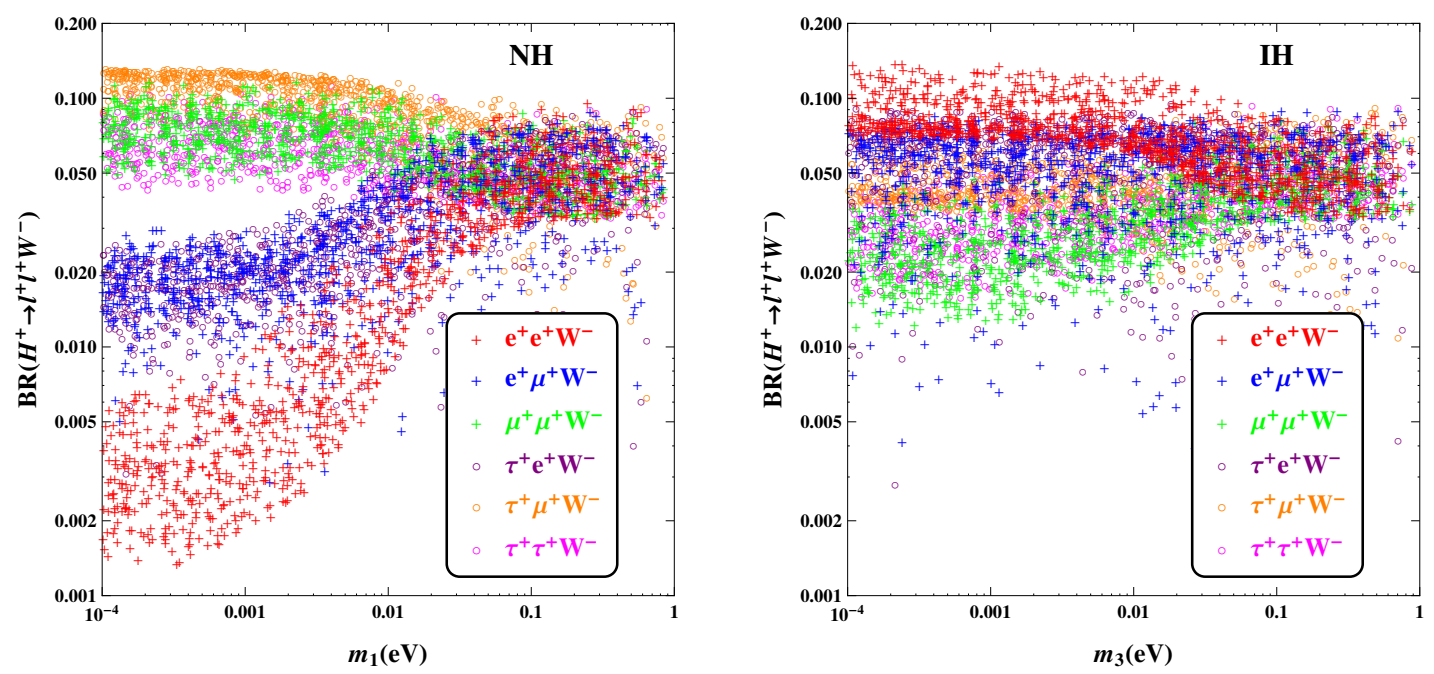

Figure 7. Branching ratios of $H^{+}$into $\ell^{+} \ell^{+} W^{-}$as a function of the lightest neutrino mass for normal (left panel) and inverted (right) hierarchy at $\left(m_{H^{+}}, m_{N_{R}}\right)=(300,200) \mathrm{GeV}$.
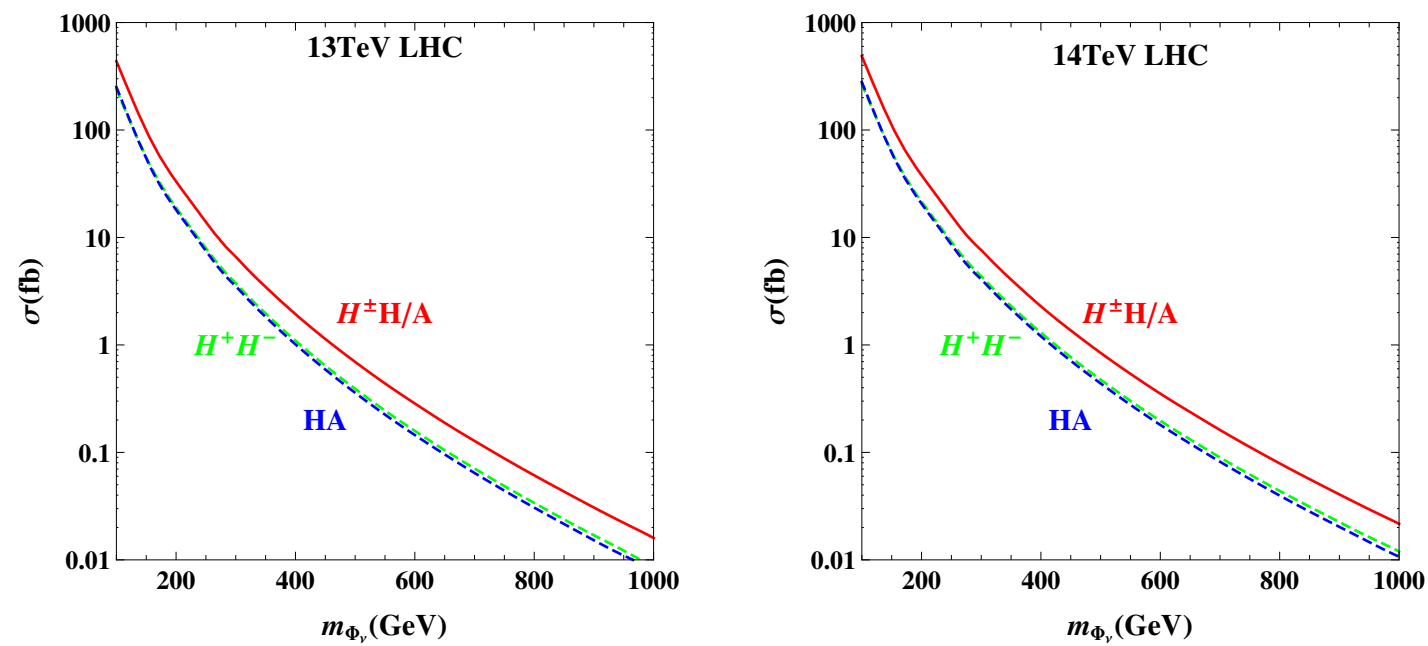

Figure 8. Cross sections for the pair and associated production of the neutrophilic scalars at 13 (14) TeV LHC as a function of their mass $m_{H^{+}}=m_{H}=m_{A}=m_{\Phi_{\nu}}$.

decaying hadronically, we have the LNV decay of the charged scalars $H^{+} \rightarrow \ell^{+} \ell^{+} j j$, which contributes to several LNV signatures at LHC. Since $\ell$ is identified as $e$ and $\mu$ at colliders, we have $\mu^{+} \mu^{+}$dominance for $\mathrm{NH}$ and $e^{+} e^{+}$dominance for IH when considering LNV signatures, which makes it possible to distinguish between the neutrino mass hierarchies.

\section{Lepton number violating signatures}

After the systematic study on the decay properties of the neutrinophilic scalars $H^{ \pm}, H$, $A$ and the heavy Majorana neutrinos $N_{R}$ in section 3, we can now investigate the LNV signatures at LHC. Our simulation procedure is as follows. We first implement the $\nu 2 \mathrm{HDM}$ into the package FeynRules [213-215] to generate the UFO [216] model file. The parton level 
signal and corresponding background events are generated with MadGraph5_aMC@NLO [217, 218] using the NNPDF2.3 [219, 220] LO parton distribution function set, and then pass through Pythia6 [221] to include showering and hadronization. Delphes3 [222, 223] is then employed for a fast detector simulation and MadAnalysis5 [224-226] for analysis. Finally, the 95\% C.L. exclusion limits are acquired by employing CheckMATE [227, 228].

We first recall that the conventional well-studied LNV signature for heavy Majorana neutrinos, $p p \rightarrow W^{ \pm} / H^{ \pm} \rightarrow \ell^{ \pm} N_{R} \rightarrow \ell^{ \pm} \ell^{ \pm} j j$, is also possible in $\nu 2 \mathrm{HDM}$, but its amplitude is suppressed by $V_{\ell N} \sim 10^{-7}$ and $v_{\nu} / v \sim 10^{-5}$ (for $v_{\nu} \sim \mathcal{O}(10 \mathrm{MeV})$ ) respectively, making the signature practically unobservable at LHC. In contrast, due to the doublet nature of the neutrinophilic scalars, they can be pair and associated produced via the Drell-Yan processes

$$
p p \rightarrow H^{+} H^{-}, H^{ \pm} H, H^{ \pm} A, H A .
$$

Their cross sections at LHC are presented in figure 8, which range from 400 to $0.01 \mathrm{fb}$ in the mass interval $100-1000 \mathrm{GeV}$ at $13 \mathrm{TeV}$, and become slightly enhanced at $14 \mathrm{TeV}$. There are many possible final states given by the decay channels of the scalars that we discussed in section 3 and the sequential decays of SM particles [173]. These channels lead to various signatures which are conventionally classified according to the multiplicities of charged leptons and jets. Among them the following three LNV signatures are most interesting and promising, and will be studied in section 4.1-4.3:

- $2 \ell^{ \pm} 4 j+\mathbb{E}_{T}$ from $H^{ \pm} H, H^{ \pm} A$ and $H A$ production,

- $3 \ell^{ \pm} 4 j+\mathbb{E}_{T}$ from $H^{ \pm} H, H^{ \pm} A$ production,

- $3 \ell^{ \pm} \ell^{\mp} 4 j$ from $H^{+} H^{-}$production,

where $\ell=e, \mu$ in our definition of a lepton for LHC signatures. In figure 9 we show the theoretical cross sections for the above LNV signatures at LHC. While the same sign dilepton (SSD) signature $2 \ell^{ \pm} 4 j+\mathbb{E}_{T}$ has the largest cross section, it is accompanied by relatively larger backgrounds. On the contrary, the four-lepton signature $3 \ell^{ \pm} \ell^{\mp} 4 j$ is clean, but its cross section is also the smallest. In between, the same sign trilepton (SST) signature $3 \ell^{ \pm} 4 j+\mathbb{E}_{T}$ seems promising, since it is nearly background free. We also notice that the cross sections for all three signals are larger for neutrino masses in IH than in NH. To illustrate the testability of the LNV signatures, we choose the following benchmark points:

$$
\begin{aligned}
& \text { BP-A : } m_{N_{R}}=200 \mathrm{GeV}, m_{\Phi_{\nu}}=300 \mathrm{GeV}, \\
& \text { BP-B : } m_{N_{R}}=300 \mathrm{GeV}, m_{\Phi_{\nu}}=400 \mathrm{GeV}, \\
& \text { BP-C : } m_{N_{R}}=400 \mathrm{GeV}, m_{\Phi_{\nu}}=500 \mathrm{GeV},
\end{aligned}
$$

which are still allowed by current constraints. The signals and backgrounds will be simulated at 13 (14) TeV with an integrated luminosity of 100 (3000) $\mathrm{fb}^{-1}$, or LHC13@100 (LHC14@3000) for short, and the corresponding exclusion limits will be derived as well. 

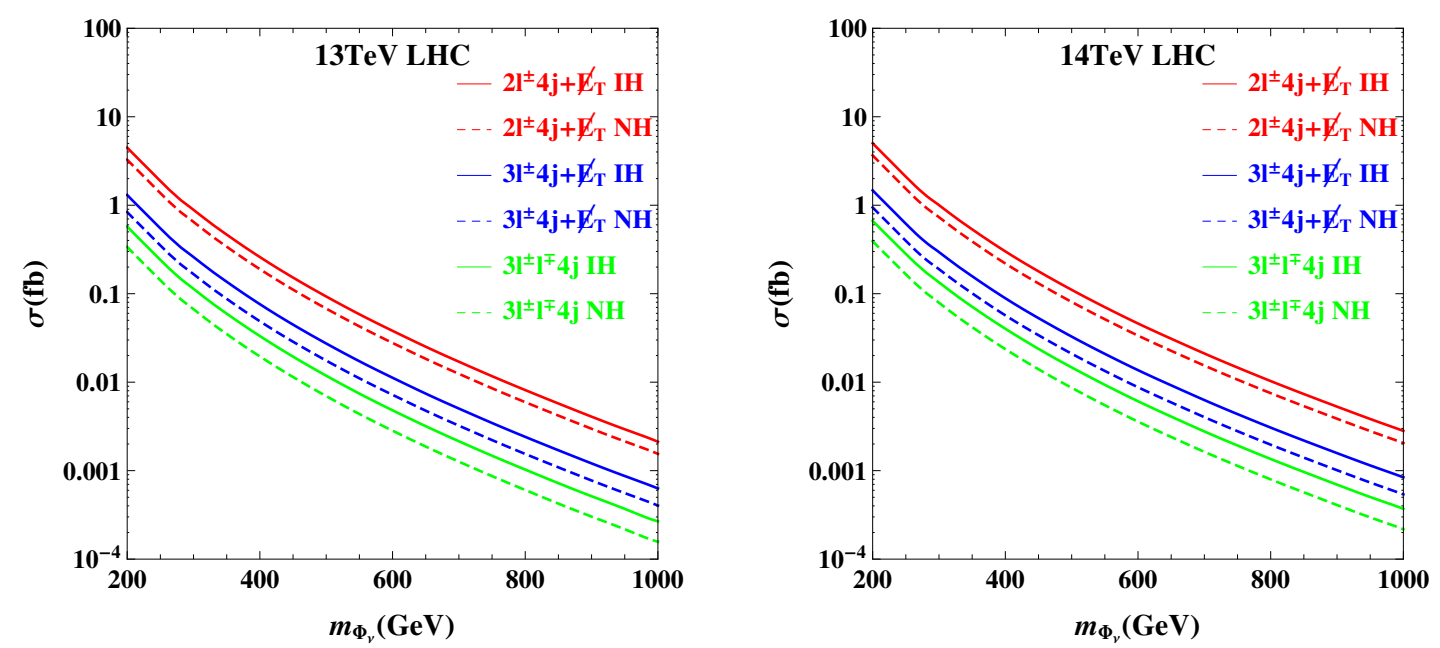

Figure 9. Cross sections of LNV signatures at 13 (14) TeV LHC.

\subsection{Dilepton signature}

The signature comes from pair and associated production of the doublet scalar $\Phi_{\nu}$ and subsequent decays:

$$
\begin{aligned}
& p p \rightarrow H^{ \pm} H / A \rightarrow \ell^{ \pm} N_{R} \nu N_{R} \rightarrow \ell^{ \pm} \ell^{ \pm} W^{\mp} \nu \nu Z / h \rightarrow \ell^{ \pm} \ell^{ \pm} j j \nu \nu j j, \\
& p p \rightarrow H A \rightarrow \nu N_{R} \nu N_{R} \rightarrow \nu \ell^{ \pm} W^{\mp} \nu \ell^{ \pm} W^{\mp} \rightarrow \nu \ell^{ \pm} j j \nu \ell^{ \pm} j j,
\end{aligned}
$$

where $\ell=e, \mu$ for collider simulations. The major sources of background are $t \bar{t} W, t \bar{t} Z$ and $W^{ \pm} W^{ \pm} W^{\mp} j j$. For the last one we use the MLM [229] matching scheme with xqcut = 25 (30) GeV for 13 (14) TeV LHC.

We show in figure 10 the distributions of the transverse momentum $p_{T}(j), p_{T}(\ell)$ and pseudorapidity $\eta(j), \eta(\ell)$ for jets and leptons, the missing transverse energy $\mathbb{E}_{T}$, and the relative distances $\Delta R_{j \ell, j j, \ell \ell}$ between leptons/jets for the SSD signature at $13 \mathrm{TeV}$ LHC (and similarly at $14 \mathrm{TeV}$ ). Note that the normalized distributions for signatures from $\mathrm{NH}$ and $\mathrm{IH}$ are the same, despite the corresponding cross sections and lepton flavor structure are different. As the backgrounds are huge compared to the sigal, we only apply some basic cuts in order to preserve the signal to the maximum extent:

$$
\begin{aligned}
& p_{T}(\ell)>10 \mathrm{GeV}, \quad p_{T}(j)>20 \mathrm{GeV}, \\
& \mathbb{E}_{T}>10 \mathrm{GeV}, \\
& |\eta(\ell)|<2.5, \\
& |\eta(j)|<5, \\
& \Delta R_{j j, \ell \ell, j \ell}>0.4 \text {. }
\end{aligned}
$$

In principle, we could tighten the cuts such as $p_{T}(\ell)>50 \mathrm{GeV}, \mathbb{E}_{T}>100 \mathrm{GeV}$ to improve the signal to background ratio. But according to our simulation for the three benchmark points in eq. (4.2), such further cuts are actually not quite efficient to improve the significance, since the signal events are also suppressed heavily. Thus we only apply the following cuts to select the desired same-sign dilepton, four-jet events:

$$
N(j)=4, N(b)=0, N\left(\ell^{ \pm}\right)=2 .
$$



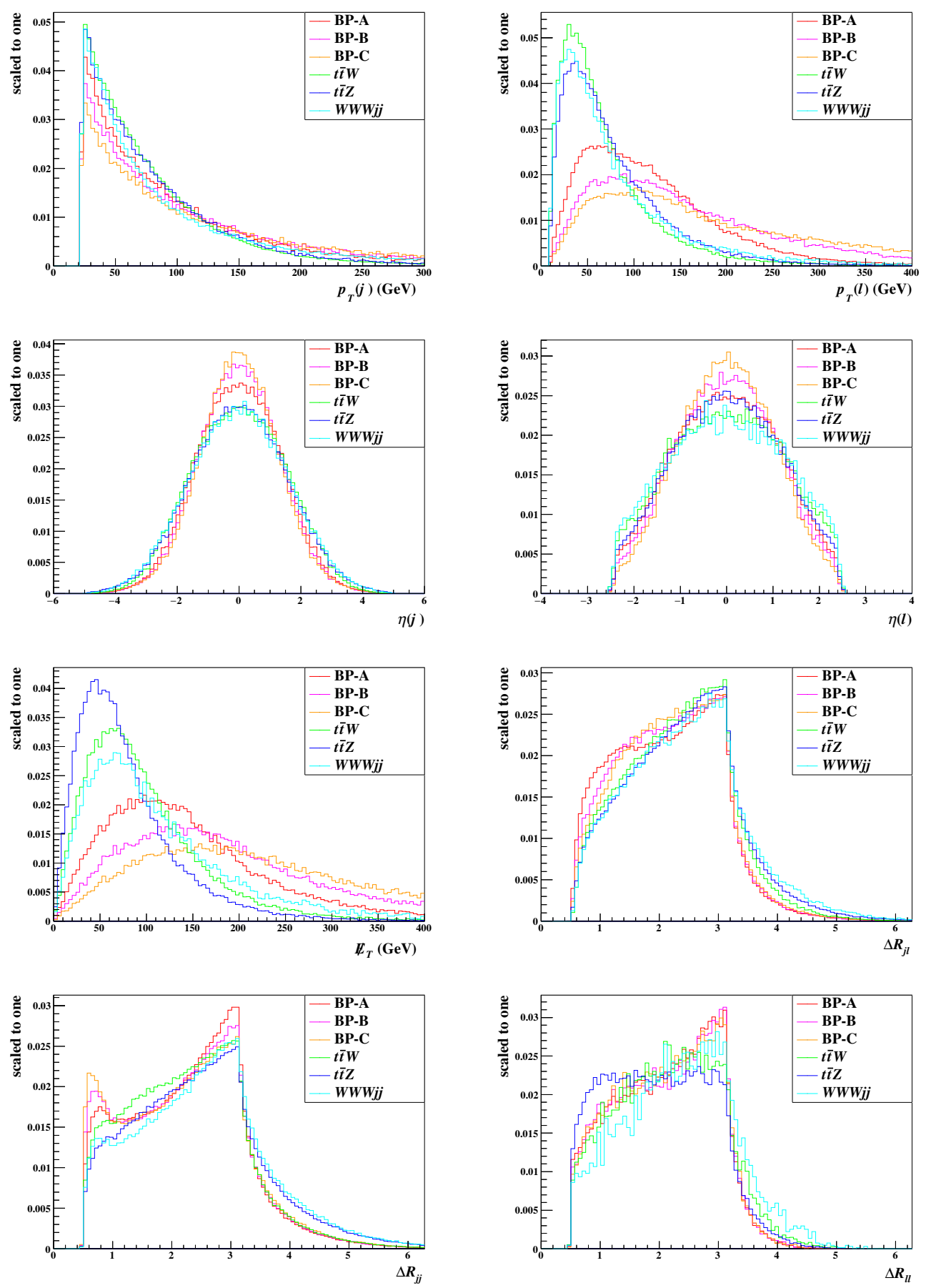

Figure 10. Distributions of transverse momenta $p_{T}(j), p_{T}(\ell)$, pseudorapidities $\eta(j), \eta(\ell)$, missing transverse energy $\mathbb{E}_{T}$, and relative distances $\Delta R_{j \ell, j j, \ell \ell}$ for the SSD signature and corresponding backgrounds at $13 \mathrm{TeV}$ LHC. 


\begin{tabular}{|c|c|c|c|c|c|c|}
\hline \multicolumn{2}{|c|}{ Channels } & Basic cuts in Eq: $(4.5)$ & $N(j)=4$ & $N(b)=0$ & $N\left(\ell^{ \pm}\right)=2$ & $S / \sqrt{S+B}$ \\
\hline \multirow{2}{*}{ BP-A } & NH & $51(1770)$ & $16(569)$ & $8.3(319)$ & $3.9(164)$ & $0.65(4.64)$ \\
& IH & $63(2425)$ & $20(779)$ & $10(436)$ & $4.9(224)$ & $0.79(6.20)$ \\
\hline \multirow{2}{*}{ BP-B } & NH & $16(566)$ & $5.0(179)$ & $2.4(90)$ & $1.3(49)$ & $0.22(1.44)$ \\
& IH & $20(775)$ & $6.1(245)$ & $3.0(124)$ & $1.6(67)$ & $0.27(1.96)$ \\
\hline \multirow{2}{*}{ BP-C } & NH & $5.8(210)$ & $1.8(65)$ & $0.87(31)$ & $0.47(17)$ & $0.08(0.51)$ \\
& IH & $7.2(288)$ & $2.2(88)$ & $1.1(43)$ & $0.58(23)$ & $0.10(0.70)$ \\
\hline \multicolumn{2}{|c|}{$t \bar{t} W$} & $1020(35023)$ & $325(10917)$ & $54(1810)$ & $18(580)$ & - \\
\multicolumn{2}{|c|}{$t \bar{t} Z$} & $1043(37909)$ & $232(8188)$ & $39(1399)$ & $2.9(172)$ & - \\
$W W W j j$ & $155(4623)$ & $43(1213)$ & $32(901)$ & $12(334)$ & - \\
\hline
\end{tabular}

Table 4. Cut-flow for the SSD signature at three benchmark points in eq. (4.2) and dominant backgrounds at LHC13@100 (LHC14@3000) for both NH and IH.
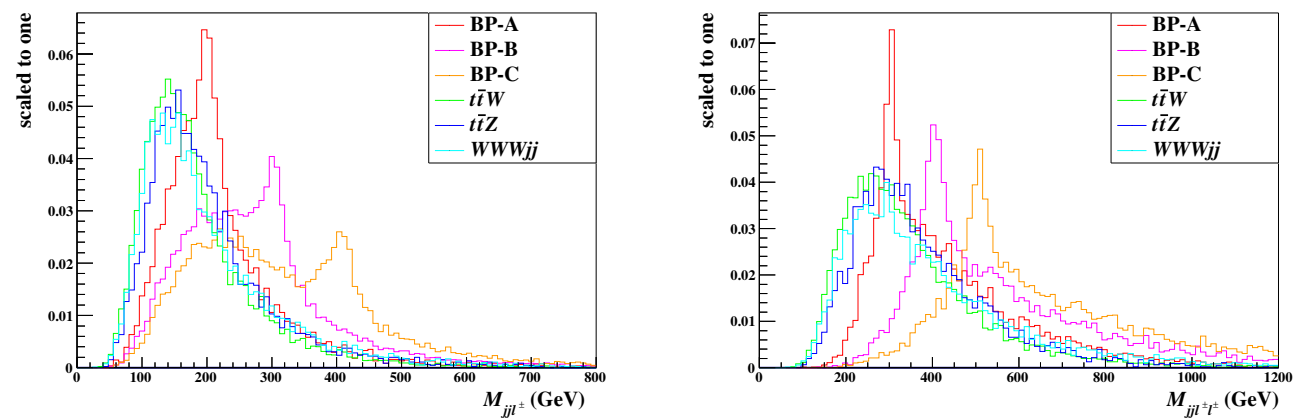

Figure 11. Left (right): reconstruction of $N_{R}\left(H^{ \pm}\right)$via invariant mass $M_{j j \ell^{ \pm}}\left(M_{j j \ell^{ \pm} \ell^{ \pm}}\right)$at $13 \mathrm{TeV}$ LHC for the SSD signature.

Here, the cut on the number of $b$-jet mainly aims to reduce the $t \bar{t} W$ and $t \bar{t} Z$ backgrounds. The identification of $b$-jets is performed with a tagging efficiency of $70 \%$, a mis-tagging rate of $10 \%$ for $c$-jets and $1 \%$ for light-flavor jets, respectively [230, 231].

In table 4 we show the cut-flow for the SSD signature at the benchmark points and the dominant backgrounds, and list the statistical significance $S / \sqrt{S+B}$ in the last column, where $S(B)$ stands for the survival number of signal (background) events after applying all cuts. As shown in figure 10, the distributions of the background and signal events (especially for BP-A) are so similar for kinematic variables like $p_{T}^{j}$ and $\Delta R_{j \ell, \ell \ell}$ that it is hard to eliminate the background without sacrificing a big part of the signal events by the naive cuts in eqs. (4.5), (4.6). As a result, the significance can barely reach $1 \sigma$ at LHC13@100. However, at LHC14@3000, we may have a chance to probe the SSD signal for BP-A, even for which a more sophisticated and efficient cut strategy is highly desired to improve the testability of this SSD signature.

For the SSD signature the decay chains $N_{R} \rightarrow \ell^{ \pm} j j$ and $H^{ \pm} \rightarrow \ell^{ \pm} \ell^{ \pm} j j$ can be used to fully reconstruct the masses of heavy neutrinos $N_{R i}$ and charged scalars $H^{ \pm}$. In figure 11, we depict the distributions for the reconstruction of $N_{R}$ and $H^{ \pm}$via the invariant mass 


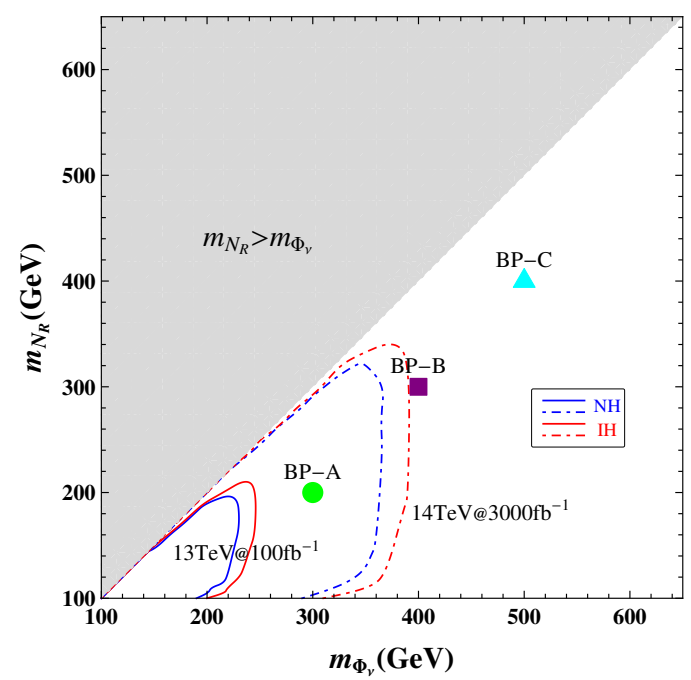

Figure 12. The expected $95 \%$ C.L. exclusion limits in the $m_{N_{R}}-m_{\Phi_{\nu}}$ plane for the SSD signature at LHC13@100 and LHC14@3000.

$M_{j j \ell^{ \pm}}$and $M_{j j \ell^{ \pm} \ell^{ \pm}}$respectively at $13 \mathrm{TeV}$ LHC after applying all cuts in eqs. (4.5), (4.6). To render the resonance peaks more discernible, we have taken advantage of the expert mode of MadAnalysis5 [224-226] so as to pick up close $j j \ell^{ \pm}$for $N_{R}$ and $j j \ell^{ \pm} \ell^{ \pm}$for $H^{ \pm}$ in the final states. We see that $N_{R}$ and $H^{ \pm}$are apparently reconstructible, although the production rate for $\mathrm{BP}-\mathrm{B}$ and $\mathrm{BP}-\mathrm{C}$ is actually too small to be detected even at LHC14@3000.

Based on the above benchmark points study, it is worthwhile to figure out the exclusion limits of the SSD signature in the $m_{N_{R}}-m_{\Phi_{\nu}}$ plane. In figure 12 we show the expected $95 \%$ C.L. exclusion limits at LHC13@100 and LHC14@3000 by employing CheckMATE [227, 228] with the cuts in eqs. (4.5), (4.6). We see that while LHC13@100 rules out a parameter region with $m_{N_{R}} \lesssim 200 \mathrm{GeV}$ and $m_{\Phi_{\nu}} \lesssim 250 \mathrm{GeV}$, LHC14@3000 can exclude a larger region up to $m_{N_{R}} \lesssim 350 \mathrm{GeV}$ and $m_{\Phi_{\nu}} \lesssim 400 \mathrm{GeV}$. Nevertheless, a compressed spectrum with $m_{\Phi_{\nu}} \approx m_{N_{R}}$ is still allowed for both $\mathrm{NH}$ and $\mathrm{IH}$, because due to the limited phase space for the $\Phi_{\nu} \rightarrow N_{R}$ decay the momentum of the leptons or $\mathbb{E}_{T}$ is too small to pass the basic cuts in eq. (4.5). We also note that the limits are more stringent for IH than NH. This originates simply from the fact that the cross section for the SSD signature in the IH case is about 1.37 times as large as the one in the $\mathrm{NH}$ case as shown in figure 9. As also shown clearly in figure 12, although all three benchmark points are beyond the reach of LHC13@100, they are either within the reach (BP-A), on the edge (BP-B) or out (BP-C) of the exclusion capability of LHC14@3000.

\subsection{Trilepton signature}

The SST signature, behaving as $3 \ell^{ \pm} 4 j+\mathbb{E}_{T}$, is the most distinct one in this $\nu 2 \mathrm{HDM}$. It originates from the associated production $H^{ \pm} H$ and $H^{ \pm} A$ :

$$
p p \rightarrow H^{ \pm} H / A \rightarrow \ell^{ \pm} N_{R} \nu N_{R} \rightarrow \ell^{ \pm} \ell^{ \pm} W^{\mp} \nu \ell^{ \pm} W^{\mp} \rightarrow 3 \ell^{ \pm} 4 j+\mathbb{E}_{T} .
$$



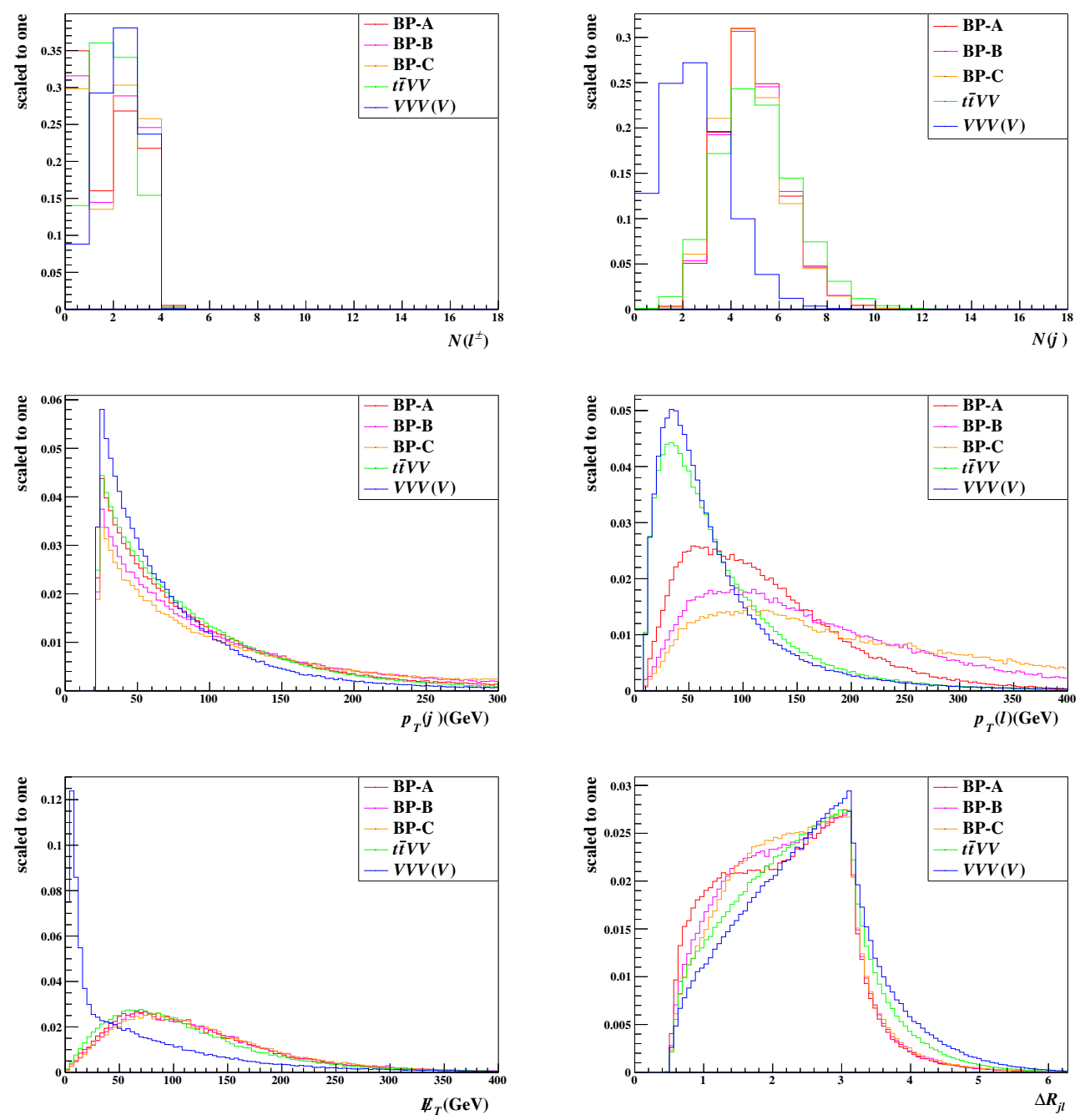

Figure 13. Distributions of numbers of same-sign leptons $N\left(\ell^{ \pm}\right)$and jets $N(j)$, transverse momenta $p_{T}(j)$ and $p_{T}(\ell)$, missing transverse energy $\mathbb{E}_{T}$, and relative distances $\Delta R_{j \ell}$ for the SST signature and corresponding backgrounds at $13 \mathrm{TeV}$ LHC.

Such a kind of signature is rather exotic in the SM as it violates the lepton number notably by three units in visible final states. The dominant backgrounds are generated from $t \bar{t} W^{ \pm} Z$ and $t \bar{t} Z Z$, where the $t \bar{t}$ pair decays semi-leptonically and the bosons decay leptonically with one lepton missed from each $Z$. The cross sections of backgrounds are extremely small, making the SST signature easier to be tested on LHC.

In figure 13 we present the distributions of the numbers of same-sign leptons $N\left(\ell^{ \pm}\right)$and jets $N(j)$, transverse momenta $p_{T}(j)$ and $p_{T}(\ell)$, missing transverse energy $\mathbb{E}_{T}$, and relative distances $\Delta R_{j \ell}$ for the SST signature at $13 \mathrm{TeV}$ LHC. The results are similar at $14 \mathrm{TeV}$. We impose the same basic cuts in eq. (4.5) as for the SSD signature. In order to separate out the signal from background, we might apply cuts such as $N\left(\ell^{ \pm}\right)=3, N(j)=4$ and $\mathbb{E}_{T}>30 \mathrm{GeV}$ to isolate the desired signature. But as mentioned above, since the 


\begin{tabular}{|c|c|c|c|c|c|}
\hline \multicolumn{2}{|c|}{ Channels } & Basic cuts in eq. (4.5) & $N\left(\ell^{ \pm}\right)=3$ & $N(j) \geq 2$ & $S / \sqrt{S+B}$ \\
\hline \multirow{2}{*}{ BP-A } & NH & $11(378)$ & $3.7(127)$ & $3.7(126)$ & $1.85(10.8)$ \\
& IH & $13(458)$ & $4.5(153)$ & $4.5(152)$ & $2.05(11.9)$ \\
\hline \multirow{2}{*}{ BP-B } & NH & $2.9(102)$ & $1.1(37)$ & $1.0(36)$ & $0.87(5.25)$ \\
& IH & $3.5(124)$ & $1.3(44)$ & $1.3(44)$ & $1.02(5.93)$ \\
\hline \multirow{2}{*}{ BP-C } & NH & $0.96(36)$ & $0.38(13)$ & $0.37(13)$ & $0.45(2.65)$ \\
& IH & $1.2(43)$ & $0.46(16)$ & $0.44(16)$ & $0.51(3.08)$ \\
\hline \multicolumn{2}{|c|}{$t \bar{t} V V$} & $1(36)$ & $0.25(8.5)$ & $0.24(8.3)$ & - \\
\multicolumn{2}{|c|}{$V V V(V)$} & $0.5(16)$ & $0.15(5)$ & $0.08(2.7)$ & - \\
\hline
\end{tabular}

Table 5. Same as table 4, but for the SST signature.
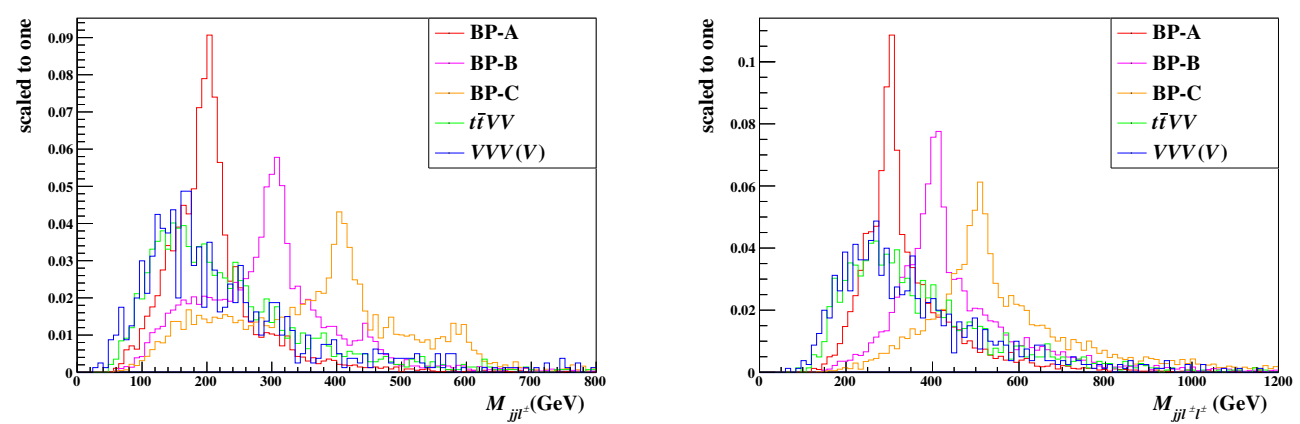

Figure 14. Same as figure 11, but for the SST signature.

background is rather clean while the signal itself has also a relatively small cross section, only few events could survive the exact selection of $3 \ell^{ \pm} 4 j+\mathbb{E}_{T}$. We thus turn to a looser selection to pick up inclusive events,

$$
N\left(\ell^{ \pm}\right)=3, N(j) \geq 2 .
$$

According to figure 13 about one third of the signal events could pass this cut. Because of this looser selection, the backgrounds from $V V V$ and $V V V V(V=W / Z)$ should be taken into account as well.

The cut-flow for the signal at the three benchmark points in eq. (4.2) in both NH and IH cases and the dominant backgrounds is presented in table 5 . The small backgrounds $t \bar{t} W^{ \pm} Z$ and $t \bar{t} Z Z$ are summed to $t \bar{t} V V$, so are $V V V$ and $V V V V$ to $V V V(V)$. At LHC13@100, only BP-A in the IH case could lead to a $2 \sigma$ excess. But at LHC14@3000, we will have a good chance to discover BP-A and BP-B, and even BP-C will result in about $2.5 \sigma(3 \sigma)$ excess for $\mathrm{NH}(\mathrm{IH})$. In figure 14, we plot the distributions in the invariant masses $M_{j j \ell^{ \pm}}$ and $M_{j j \ell^{ \pm} \ell^{ \pm}}$for the reconstruction of the $N_{R}$ and $H^{ \pm}$particles at $13 \mathrm{TeV}$ LHC.

Based on the cuts in eqs. (4.5), (4.8) we now acquire the expected 95\% C.L. exclusion limits at LHC13@100 and LHC14@300 by scanning in the $m_{N_{R}}-m_{\Phi_{\nu}}$ plane. Our results are presented in figure 15. For instance, at LHC13@100, $m_{\Phi_{\nu}}$ could be excluded up to 


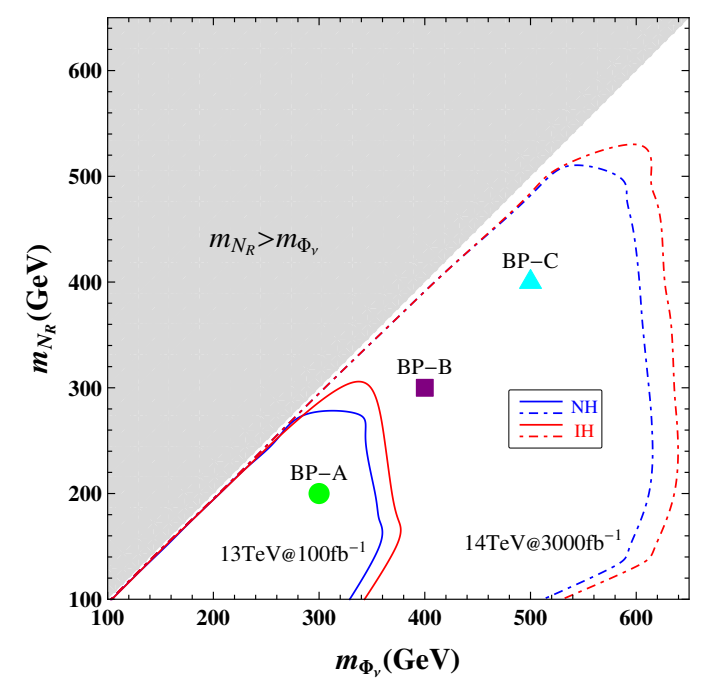

Figure 15. Same as figure 12, but for the SST signature.

about $350 \mathrm{GeV}$ for $\mathrm{NH}$ or $380 \mathrm{GeV}$ for $\mathrm{IH}$ at $m_{N_{R}} \sim 150 \mathrm{GeV}$, or conversely, $m_{N_{R}}$ could be excluded up to about $280 \mathrm{GeV}$ for $\mathrm{NH}$ or $305 \mathrm{GeV}$ for $\mathrm{IH}$ at $m_{\Phi_{\nu}} \sim 330 \mathrm{GeV}$. With higher integrated luminosity at LHC14@3000, the exclusion limit would extend to $m_{\Phi_{\nu}} \lesssim$ $600 \mathrm{GeV}, m_{N_{R}} \lesssim 500 \mathrm{GeV}$ for $\mathrm{NH}$, and even higher for IH, i.e., $m_{\Phi_{\nu}} \lesssim 640 \mathrm{GeV}, m_{N_{R}} \lesssim$ $530 \mathrm{GeV}$. It is evident that LHC13@100 (LHC14@3000) will be capable of excluding BP-A (BP-B and BP-C) through the SST signature.

\subsection{Four-lepton signature}

Finally we study the most exotic signature involving four leptons, as a result of the decay chains:

$$
p p \rightarrow H^{+} H^{-} \rightarrow \ell^{+} N_{R} \ell^{-} N_{R} \rightarrow \ell^{+} W^{\mp} \ell^{ \pm} \ell^{-} W^{\mp} \ell^{ \pm} \rightarrow 3 \ell^{ \pm} \ell^{\mp} 4 j .
$$

This is also the only case that involves two charged scalars in the intermediate state. The major sources of background are $t \bar{t} h, t \bar{t} V, t \bar{t} t \bar{t}, t \bar{t} V V$, with $V=W^{ \pm}, Z$. We found that the kinematical distributions for the four-lepton signature are similar to those for the SST signature. We impose the same basic cuts in eq. (4.5) but of course drop the cut on $\mathbb{E}_{T}$, because no invisible particles are involved now. In addition, we apply the specific cuts for the four-lepton signature:

$$
N(j)=4, N\left(\ell^{ \pm}\right)=3, N\left(\ell^{\mp}\right)=1 .
$$

No further cuts are required, since we are practically background free at this stage as will be shown below.

In table 6 we show the cut-flow for the four-lepton signature at the three benchmark points in eq. (4.2) as well as for the dominant backgrounds. We notice that the backgrounds become zero after the selection cuts in eqs. (4.5), (4.10), and this increases the feasibility of the four-lepton signature remarkably. However, we should not forget that this signal is also the weakest among the three studied in this work. At LHC13@100, this signature is 


\begin{tabular}{|c|c|c|c|c|c|}
\hline \multicolumn{2}{|c|}{ Channels } & Basic cuts & $N(j)=4$ & $\begin{array}{c}N\left(\ell^{ \pm}\right)=3 \\
N\left(\ell^{\mp}\right)=1\end{array}$ & $S / \sqrt{S+B}$ \\
\hline \multirow{2}{*}{ BP-A } & NH & $23(805)$ & $5.6(195)$ & $0.74(29)$ & $0.86(5.39)$ \\
& $\mathrm{IH}$ & $40(1404)$ & $9.8(340)$ & $1.3(43)$ & $1.14(6.56)$ \\
\hline \multirow{2}{*}{ BP-B } & $\mathrm{NH}$ & $7.2(242)$ & $1.7(58)$ & $0.27(8.7)$ & $0.52(2.95)$ \\
& $\mathrm{IH}$ & $13(422)$ & $3.0(102)$ & $0.47(15)$ & $0.69(3.87)$ \\
\hline \multirow{2}{*}{ BP-C } & $\mathrm{NH}$ & $2.4(89)$ & $0.58(21)$ & $0.09(3.2)$ & $0.30(1.79)$ \\
& $\mathrm{IH}$ & $4.2(155)$ & $1.0(37)$ & $0.16(5.6)$ & $0.40(2.37)$ \\
\hline \multicolumn{2}{|c|}{$t \bar{t} h$} & $403(33431)$ & $36(2869)$ & $0(0)$ & - \\
$t \bar{t} t \bar{t}$ & $125(11316)$ & $11(971)$ & $0(0)$ & - \\
$t \bar{t} V$ & $6043(488674)$ & $538(41943)$ & $0(0)$ & - \\
\hline
\end{tabular}

Table 6. Same as table 4, but for the four-lepton signature.
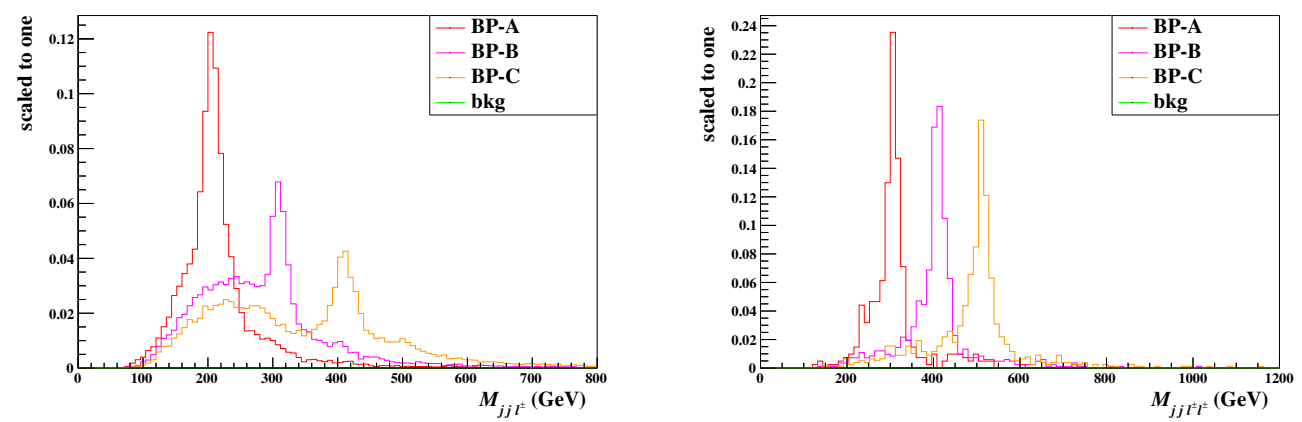

Figure 16. Same as figure 11, but for the four-lepton signature.

undetectable even for BP-A. At LHC14@3000, BP-A will have a 5.39 (6.56) $\sigma$ significance in the case of $\mathrm{NH}(\mathrm{IH})$, while BP-B can only lead to a 2.95 (3.87) $\sigma$ significance. To speak roughly about the prospect to observe such signatures at LHC, the four-lepton signature lies in between the dilepton and trilepton signatures. Adopting the same method as for the other two signatures, we reconstruct in figure 16 the heavy Majorana neutrinos $N_{R}$ and charged scalars $H^{ \pm}$via the distributions in the invariant masses $M_{j j l^{ \pm}}$and $M_{j j l^{ \pm} l^{ \pm}}$ respectively. Although the peaks in the normalized distributions are most remarkable among the three, the number of signal events is also the least.

The expected $95 \%$ C.L. exclusion limits for the four-lepton signature are shown in figure 17. The region with $m_{N_{R}} \lesssim 250 \mathrm{GeV}$ and $m_{\Phi_{\nu}} \lesssim 300 \mathrm{GeV}$ could be excluded at LHC13@100, while LHC14@3000 could reach $m_{N_{R}} \lesssim 450 \mathrm{GeV}$ and $m_{\Phi_{\nu}} \lesssim 500 \mathrm{GeV}$. Interestingly, we find that BP-A for IH but not for NH could be excluded by LHC13@100, while BP-B would be fully covered by LHC14@3000 for both NH and IH cases.

\section{Conclusion}

The neutrinophilic two-Higgs-doublet model provides a natural way to generate tiny neutrino mass with $\mathrm{TeV}$ scale Majorana neutrinos $N_{R}$ and scalars $\Phi_{\nu}$, and results in interesting 


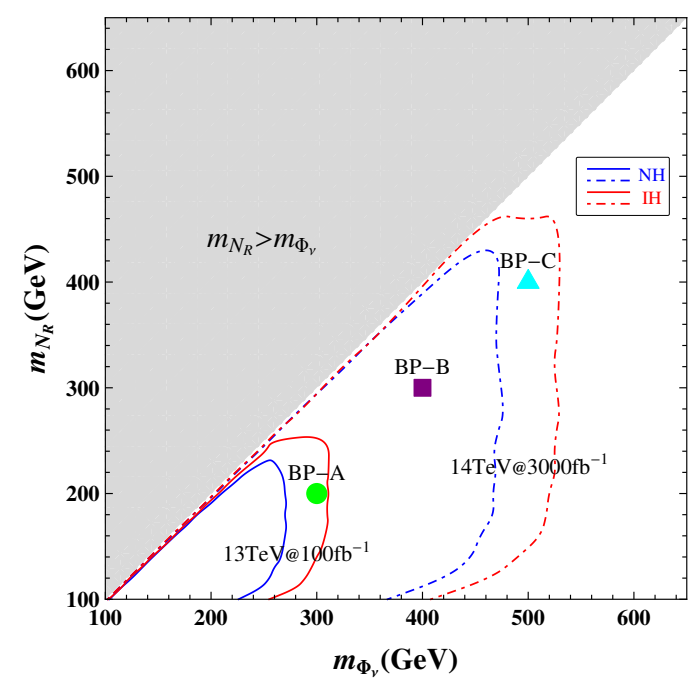

Figure 17. Same as figure 12, but for the four-lepton signature.

phenomena such as LFV processes and LNV signatures at LHC. We found that the LFV processes of the charged leptons mediated by the Yukawa coupling $\bar{L} \widetilde{\Phi}_{\nu} N_{R}$ can be within the reach of current experiments. Using the most stringent constraint on the decay $\mu \rightarrow e \gamma$, we derived a combined tight bound on the $\Phi_{\nu}$ mass and VEV, $m_{H^{+}} v_{\nu} \gtrsim 600 \mathrm{GeV} \cdot \mathrm{MeV}$, for $N_{R}$ of a few hundreds GeV.

Our work has been focused on the LNV signatures from heavy Majorana neutrinos at LHC. To achieve this, we systematically investigated the decay properties of the neutrinophilic scalars $H^{ \pm}, H, A$ and heavy Majorana neutrinos $N_{R}$ for the mass order $m_{N_{R}}<m_{\Phi_{\nu}}$. Our results show that there is strong correlation between the neutrino mass hierarchy and the flavor fraction of charged leptons in the decays of $H^{ \pm}$and $N_{R}$. In particular, we expect $\mu^{ \pm} \mu^{ \pm}\left(e^{ \pm} e^{ \pm}\right)$dominance for NH (IH) in the LNV decay chain $H^{ \pm} \rightarrow \ell^{ \pm} N_{R} \rightarrow \ell^{ \pm} \ell^{ \pm} j j(\ell=e, \mu)$. In addition, the production rate for LNV signatures is larger in the case of $\mathrm{IH}$ than $\mathrm{NH}$, making the physics signals in the former case more promising to be detected at LHC.

When the new scalars are heavier than the new neutrinos, they are first produced at LHC via the Drell-Yan processes and then cascade decay into the new neutrinos $H^{ \pm} \rightarrow$ $\ell^{ \pm} N_{R}, H / A \rightarrow \nu N_{R}$ and the SM particles $N_{R} \rightarrow \ell^{ \pm} W^{\mp}, \nu Z, \nu h$. This results in three kinds of LNV signatures: $2 \ell^{ \pm} 4 j+\mathbb{E}_{T}, 3 \ell^{ \pm} 4 j+\mathbb{E}_{T}$, and $3 \ell^{ \pm} \ell^{\mp} 4 j$. To illustrate the testability of such LNV signatures at LHC, we worked with three benchmark points (BP-A, -B, -C, for short), $\left(m_{N_{R}}, m_{\Phi_{\nu}}\right)=(200,300),(300,400),(400,500) \mathrm{GeV}$, and performed detailed simulations. Our simulation results are recapitulated in table 7 . We found that the SST signature is the most promising among the three. Although at LHC13@100 it is hard to observe excess events of the SST signature even for BP-A, we can discover BP-A and BP-B from the SST signature at LHC14@3000. Furthermore, BP-C with both scalars and neutrinos much heavier can also lead to about $2.5 \sigma(3 \sigma)$ excess for NH $(\mathrm{IH})$ at LHC14@3000. With such high integrated luminosity there is also a good chance to probe the SSD and four-lepton signatures for BP-A. Conversely, if no excess is observed, the mass region of 


\begin{tabular}{|c|c|c|c|c|}
\hline \multicolumn{2}{|c|}{ LNV signatures } & $2 \ell^{ \pm} 4 j \mathbb{E}_{T}$ & $3 \ell^{ \pm} 4 j \mathbb{E}_{T}$ & $3 \ell^{ \pm} \ell^{\mp} 4 j$ \\
\hline \multirow{2}{*}{$\mathrm{BP}-\mathrm{A}$} & $\mathrm{NH}$ & $0.65(4.64)$ & $1.85(10.8)$ & $0.86(5.39)$ \\
& $\mathrm{IH}$ & $0.79(6.20)$ & $2.05(11.9)$ & $1.14(6.56)$ \\
\hline \multirow{2}{*}{$\mathrm{BP}-\mathrm{B}$} & $\mathrm{NH}$ & $0.22(1.44)$ & $0.87(5.25)$ & $0.52(2.95)$ \\
& $\mathrm{IH}$ & $0.27(1.96)$ & $1.02(5.93)$ & $0.69(3.87)$ \\
\hline \multirow{2}{*}{$\mathrm{BP}-\mathrm{C}$} & $\mathrm{NH}$ & $0.08(0.51)$ & $0.45(2.65)$ & $0.30(1.79)$ \\
& $\mathrm{IH}$ & $0.10(0.70)$ & $0.51(3.08)$ & $0.40(2.37)$ \\
\hline \multirow{2}{*}{$M_{N_{R}}$} & $\mathrm{NH}$ & $200(320)$ & $280(500)$ & $230(420)$ \\
& $\mathrm{IH}$ & $215(340)$ & $305(530)$ & $250(460)$ \\
\hline \multirow{2}{*}{$M_{\Phi_{\nu}}$} & $\mathrm{NH}$ & $230(360)$ & $350(600)$ & $270(470)$ \\
& $\mathrm{IH}$ & $250(390)$ & $380(640)$ & $310(530)$ \\
\hline
\end{tabular}

Table 7. Summary of statistical significance for three types of signatures at three benchmark points and of approximate lower bounds on new particle masses to be expected at LHC13@100 (LHC14@3000) for neutrino masses in either NH or IH.

$m_{N_{R}} \lesssim 300 \mathrm{GeV}$ and $m_{\Phi_{\nu}} \lesssim 350 \mathrm{GeV}$ will be excluded by LHC13@100, and the excluded region will be extended at LHC14@3000 to $m_{N_{R}} \lesssim 500 \mathrm{GeV}$ and $m_{\Phi_{\nu}} \lesssim 600 \mathrm{GeV}$ by the SST signature.

\section{Acknowledgments}

This work was supported in part by the Grants No. NSFC-11575089, No. NSFC-11025525 and by the CAS Center for Excellence in Particle Physics (CCEPP). Part of numerical analysis was done with the HPC Cluster of SKLTP/ITP-CAS.

Open Access. This article is distributed under the terms of the Creative Commons Attribution License (CC-BY 4.0), which permits any use, distribution and reproduction in any medium, provided the original author(s) and source are credited.

\section{References}

[1] S. Weinberg, Baryon and Lepton Nonconserving Processes, Phys. Rev. Lett. 43 (1979) 1566 [INSPIRE].

[2] E. Ma, Pathways to naturally small neutrino masses, Phys. Rev. Lett. 81 (1998) 1171 [hep-ph/9805219] [INSPIRE].

[3] P. Minkowski, $\mu \rightarrow$ er at a rate of one out of $10^{9}$ muon decays?, Phys. Lett. B 67 (1977) 421 [INSPIRE].

[4] T. Yanagida, Horizontal symmetry and masses of neutrinos, in the proceedings of the Workshop on unified theory and baryon number in the universe, O. Sawada and A. Sugamoto eds., KEK, Tsukuba, Japan (1979). 
[5] M. Gell-Mann, P. Ramond, R. Slansky, Complex spinors and unified theories, in Supergravity, D.Z. Freedman and P.van Nieuwenhuizen eds., North Holland, Amsterdam, The Netherlands (1979).

[6] S.L. Glashow, The future of elementary particle physics, in Quarks and leptons, Cargèse lectures, M. Lévy et al. eds., Plenum Press, New York, U.S.A. (1980).

[7] R.N. Mohapatra and G. Senjanović, Neutrino mass and spontaneous parity violation, Phys. Rev. Lett. 44 (1980) 912 [INSPIRE].

[8] M. Magg and C. Wetterich, Neutrino mass problem and gauge hierarchy, Phys. Lett. B 94 (1980) 61 [INSPIRE].

[9] T.P. Cheng and L.-F. Li, Neutrino masses, mixings and oscillations in $\mathrm{SU}(2) \times \mathrm{U}(1)$ models of electroweak interactions, Phys. Rev. D 22 (1980) 2860 [inSPIRE].

[10] J. Schechter and J.W.F. Valle, Neutrino masses in $\mathrm{SU}(2) \times \mathrm{U}(1)$ theories, Phys. Rev. D 22 (1980) 2227 [INSPIRE].

[11] G. Lazarides, Q. Shafi and C. Wetterich, Proton lifetime and fermion masses in an $\mathrm{SO}(10)$ model, Nucl. Phys. B 181 (1981) 287 [InSPIRE].

[12] R.N. Mohapatra and G. Senjanović, Neutrino masses and mixings in gauge models with spontaneous parity violation, Phys. Rev. D 23 (1981) 165 [InSPIRE].

[13] R. Foot, H. Lew, X.G. He and G.C. Joshi, Seesaw neutrino masses induced by a triplet of leptons, Z. Phys. C 44 (1989) 441 [INSPIRE].

[14] D. Wyler and L. Wolfenstein, Massless neutrinos in left-right symmetric models, Nucl. Phys. B 218 (1983) 205 [INSPIRE].

[15] R.N. Mohapatra and J.W.F. Valle, Neutrino mass and baryon number nonconservation in superstring models, Phys. Rev. D 34 (1986) 1642 [INSPIRE].

[16] E. Ma, Lepton number nonconservation in $E_{6}$ superstring models, Phys. Lett. B 191 (1987) 287 [INSPIRE].

[17] E.K. Akhmedov, M. Lindner, E. Schnapka and J.W.F. Valle, Left-right symmetry breaking in NJLS approach, Phys. Lett. B 368 (1996) 270 [hep-ph/9507275] [INSPIRE].

[18] E.K. Akhmedov, M. Lindner, E. Schnapka and J.W.F. Valle, Dynamical left-right symmetry breaking, Phys. Rev. D 53 (1996) 2752 [hep-ph/9509255] [INSPIRE].

[19] M. Malinsky, J.C. Romao and J.W.F. Valle, Novel supersymmetric $\mathrm{SO}(10)$ seesaw mechanism, Phys. Rev. Lett. 95 (2005) 161801 [hep-ph/0506296] [INSPIRE].

[20] K.S. Babu and C.N. Leung, Classification of effective neutrino mass operators, Nucl. Phys. B 619 (2001) 667 [hep-ph/0106054] [INSPIRE].

[21] K.S. Babu, S. Nandi and Z. Tavartkiladze, New mechanism for neutrino mass generation and triply charged Higgs bosons at the LHC, Phys. Rev. D 80 (2009) 071702 [arXiv:0905.2710] [INSPIRE].

[22] F. Bonnet, D. Hernandez, T. Ota and W. Winter, Neutrino masses from higher than D=5 effective operators, JHEP 10 (2009) 076 [arXiv:0907.3143] [INSPIRE].

[23] I. Picek and B. Radovcic, Novel TeV-scale seesaw mechanism with Dirac mediators, Phys. Lett. B 687 (2010) 338 [arXiv:0911.1374] [INSPIRE].

[24] Y. Liao, Unique neutrino mass operator at any mass dimension, Phys. Lett. B 694 (2011) 346 [arXiv:1009.1692] [INSPIRE].

[25] Y. Liao, Cascade seesaw for tiny neutrino mass, JHEP 06 (2011) 098 [arXiv:1011.3633] [INSPIRE]. 
[26] B. Ren, K. Tsumura and X.-G. He, A Higgs quadruplet for type III seesaw and implications for $\mu \rightarrow e \gamma$ and $\mu-e$ conversion, Phys. Rev. D 84 (2011) 073004 [arXiv:1107.5879] [INSPIRE].

[27] K. Kumericki, I. Picek and B. Radovcic, Exotic seesaw-motivated heavy leptons at the LHC, Phys. Rev. D 84 (2011) 093002 [arXiv:1106.1069] [INSPIRE].

[28] K. Kumericki, I. Picek and B. Radovcic, TeV-scale seesaw with quintuplet fermions, Phys. Rev. D 86 (2012) 013006 [arXiv:1204.6599] [InSPIRE].

[29] I. Picek and B. Radovcic, Enhancement of $h \rightarrow \gamma \gamma$ by seesaw-motivated exotic scalars, Phys. Lett. B 719 (2013) 404 [arXiv: 1210.6449] [INSPIRE].

[30] K.L. McDonald, Minimal tree-level seesaws with a heavy intermediate fermion, JHEP 07 (2013) 020 [arXiv: 1303.4573] [inSPIRE].

[31] S.S.C. Law and K.L. McDonald, Generalized inverse seesaw mechanisms, Phys. Rev. D 87 (2013) 113003 [arXiv:1303.4887] [inSPIRE].

[32] A. Zee, A theory of lepton number violation, neutrino majorana mass and oscillation, Phys. Lett. B 93 (1980) 389 [Erratum ibid. B 95 (1980) 461] [INSPIRE].

[33] L. Wolfenstein, A theoretical pattern for neutrino oscillations, Nucl. Phys. B 175 (1980) 93 [INSPIRE].

[34] A. Zee, Quantum numbers of Majorana neutrino masses, Nucl. Phys. B 264 (1986) 99 [INSPIRE].

[35] K.S. Babu, Model of 'calculable' Majorana neutrino masses, Phys. Lett. B 203 (1988) 132 [INSPIRE].

[36] K.S. Babu and E. Ma, Natural hierarchy of radiatively induced Majorana neutrino masses, Phys. Rev. Lett. 61 (1988) 674 [INSPIRE].

[37] E. Ma, Verifiable radiative seesaw mechanism of neutrino mass and dark matter, Phys. Rev. D 73 (2006) 077301 [hep-ph/0601225] [INSPIRE].

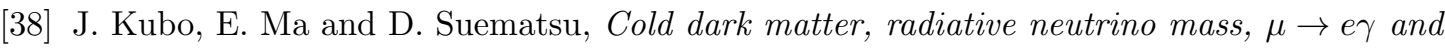
neutrinoless double beta decay, Phys. Lett. B 642 (2006) 18 [hep-ph/0604114] [INSPIRE].

[39] P.-H. Gu and U. Sarkar, Radiative neutrino mass, dark matter and leptogenesis, Phys. Rev. D 77 (2008) 105031 [arXiv:0712.2933] [INSPIRE].

[40] D. Aristizabal Sierra, J. Kubo, D. Restrepo, D. Suematsu and O. Zapata, Radiative seesaw: warm dark matter, collider and lepton flavour violating signals, Phys. Rev. D 79 (2009) 013011 [arXiv: 0808.3340] [INSPIRE].

[41] E. Ma and D. Suematsu, Fermion triplet dark matter and radiative neutrino mass, Mod. Phys. Lett. A 24 (2009) 583 [arXiv:0809.0942] [InSPIRE].

[42] S. Kanemura, T. Nabeshima and H. Sugiyama, Neutrino masses from loop-induced Dirac Yukawa couplings, Phys. Lett. B 703 (2011) 66 [arXiv:1106.2480] [INSPIRE].

[43] S. Kanemura, O. Seto and T. Shimomura, Masses of dark matter and neutrino from TeV scale spontaneous $U(1)_{B-L}$ breaking, Phys. Rev. D 84 (2011) 016004 [arXiv:1101.5713] [INSPIRE].

[44] S. Kanemura and H. Sugiyama, Dark matter and a suppression mechanism for neutrino masses in the Higgs triplet model, Phys. Rev. D 86 (2012) 073006 [arXiv:1202.5231] [INSPIRE].

[45] F. Bonnet, M. Hirsch, T. Ota and W. Winter, Systematic study of the D $=5$ Weinberg operator at one-loop order, JHEP 07 (2012) 153 [arXiv:1204.5862] [INSPIRE]. 
[46] H. Okada and T. Toma, Fermionic dark matter in radiative inverse seesaw model with $\mathrm{U}(1)_{B-L}$, Phys. Rev. D 86 (2012) 033011 [arXiv:1207.0864] [InSPIRE].

[47] P.S.B. Dev and A. Pilaftsis, Minimal radiative neutrino mass mechanism for inverse seesaw models, Phys. Rev. D 86 (2012) 113001 [arXiv:1209.4051] [INSPIRE].

[48] D. Schmidt, T. Schwetz and T. Toma, Direct detection of leptophilic dark matter in a model with radiative neutrino masses, Phys. Rev. D 85 (2012) 073009 [arXiv:1201.0906] [INSPIRE].

[49] M. Hirsch, R.A. Lineros, S. Morisi, J. Palacio, N. Rojas and J.W.F. Valle, WIMP dark matter as radiative neutrino mass messenger, JHEP 10 (2013) 149 [arXiv:1307.8134] [INSPIRE].

[50] S.S.C. Law and K.L. McDonald, A class of inert $N$-tuplet models with radiative neutrino mass and dark matter, JHEP 09 (2013) 092 [arXiv: 1305.6467] [INSPIRE].

[51] S. Kanemura, T. Matsui and H. Sugiyama, Loop suppression of Dirac neutrino mass in the neutrinophilic two Higgs doublet model, Phys. Lett. B 727 (2013) 151 [arXiv:1305.4521] [INSPIRE].

[52] D. Restrepo, O. Zapata and C.E. Yaguna, Models with radiative neutrino masses and viable dark matter candidates, JHEP 11 (2013) 011 [arXiv:1308.3655] [INSPIRE].

[53] V. Brdar, I. Picek and B. Radovcic, Radiative neutrino mass with scotogenic scalar triplet, Phys. Lett. B 728 (2014) 198 [arXiv: 1310.3183] [InSPIRE].

[54] H. Okada and K. Yagyu, Radiative generation of lepton masses with the $\mathrm{U}(1)^{\prime}$ gauge symmetry, Phys. Rev. D 90 (2014) 035019 [arXiv:1405.2368] [INSPIRE].

[55] W. Wang and Z.-L. Han, Radiative linear seesaw model, dark matter and $\mathrm{U}(1)_{B-L}$, Phys. Rev. D 92 (2015) 095001 [arXiv: 1508.00706] [INSPIRE].

[56] R. Longas, D. Portillo, D. Restrepo and O. Zapata, The inert Zee model, JHEP 03 (2016) 162 [arXiv:1511.01873] [INSPIRE].

[57] R. Adhikari, D. Borah and E. Ma, New U(1) gauge model of radiative lepton masses with sterile neutrino and dark matter, Phys. Lett. B 755 (2016) 414 [arXiv:1512.05491] [INSPIRE].

[58] H. Okada and Y. Orikasa, Radiative neutrino model with an inert triplet scalar, Phys. Rev. D 94 (2016) 055002 [arXiv: 1512.06687] [InSPIRE].

[59] A. Ibarra, C.E. Yaguna and O. Zapata, Direct detection of fermion dark matter in the radiative seesaw model, Phys. Rev. D 93 (2016) 035012 [arXiv:1601.01163] [INSPIRE].

[60] R. Ding, Z.-L. Han, Y. Liao and X.-D. Ma, Interpretation of $750 \mathrm{GeV}$ diphoton excess at LHC in singlet extension of color-octet neutrino mass model, Eur. Phys. J. C 76 (2016) 204 [arXiv: 1601.02714] [INSPIRE].

[61] A. Ahriche, K.L. McDonald, S. Nasri and I. Picek, A critical analysis of one-loop neutrino mass models with minimal dark matter, Phys. Lett. B 757 (2016) 399 [arXiv:1603.01247] [INSPIRE].

[62] C. Kownacki and E. Ma, Gauge U(1) dark symmetry and radiative light fermion masses, Phys. Lett. B 760 (2016) 59 [arXiv: 1604.01148] [INSPIRE].

[63] E. Ma, N. Pollard, O. Popov and M. Zakeri, Gauge B - L model of radiative neutrino mass with multipartite dark matter, Mod. Phys. Lett. A 31 (2016) 1650163 [arXiv:1605.00991] [INSPIRE].

[64] T. Nomura, H. Okada and Y. Orikasa, $\mathrm{SU}(2)_{L}$ septet scalar linking to a radiative neutrino model, Phys. Rev. D 94 (2016) 055012 [arXiv: 1605. 02601] [INSPIRE]. 
[65] T. Nomura and H. Okada, Radiatively induced quark and lepton mass model, Phys. Lett. B 761 (2016) 190 [arXiv: 1606.09055] [INSPIRE].

[66] P.-H. Gu, E. Ma and U. Sarkar, Connecting radiative neutrino mass, neutron-antineutron oscillation, proton decay and leptogenesis through dark matter, Phys. Rev. D 94 (2016) 111701 [arXiv: 1608.02118] [INSPIRE].

[67] D. Borah and A. Dasgupta, Common origin of neutrino mass, dark matter and Dirac leptogenesis, JCAP 12 (2016) 034 [arXiv: 1608.03872] [INSPIRE].

[68] S.-Y. Guo, Z.-L. Han and Y. Liao, Testing the type-II radiative seesaw model: From dark matter detection to LHC signatures, Phys. Rev. D 94 (2016) 115014 [arXiv:1609.01018] [INSPIRE].

[69] T. Nomura, H. Okada and Y. Orikasa, Radiative neutrino model with $\mathrm{SU}(2)_{L}$ triplet fields, Phys. Rev. D 94 (2016) 115018 [arXiv:1610.04729] [InSPIRE].

[70] W. Wang and Z.-L. Han, Naturally small Dirac neutrino mass with intermediate $\mathrm{SU}(2)_{l}$ multiplet fields, arXiv:1611.03240 [INSPIRE].

[71] L.T. Hue, A.B. Arbuzov, N.T.K. Ngan and H.N. Long, Probing neutrino and Higgs sectors in $\mathrm{SU}(2)_{1} \times \mathrm{SU}(2)_{2} \times \mathrm{U}(1)_{Y}$ model with lepton-flavor non-universality, arXiv:1611.06801 [INSPIRE].

[72] E. Ma, $Z(3)$ dark matter and two-loop neutrino mass, Phys. Lett. B 662 (2008) 49 [arXiv:0708.3371] [INSPIRE].

[73] S. Kanemura, T. Nabeshima and H. Sugiyama, TeV-scale seesaw with loop-induced Dirac mass term and dark matter from $U(1)_{B-L}$ gauge symmetry breaking, Phys. Rev. D 85 (2012) 033004 [arXiv:1111.0599] [INSPIRE].

[74] S. Baek, P. Ko, H. Okada and E. Senaha, Can Zee-Babu model implemented with scalar dark matter explain both Fermi/LAT $130 \mathrm{GeV}$-ray excess and neutrino physics?, JHEP 09 (2014) 153 [arXiv: 1209.1685] [INSPIRE].

[75] Y. Kajiyama, H. Okada and K. Yagyu, Two loop radiative seesaw model with inert triplet scalar field, Nucl. Phys. B 874 (2013) 198 [arXiv: 1303.3463] [INSPIRE].

[76] Y. Kajiyama, H. Okada and T. Toma, Multicomponent dark matter particles in a two-loop neutrino model, Phys. Rev. D 88 (2013) 015029 [arXiv: 1303.7356] [INSPIRE].

[77] S. Baek, H. Okada and T. Toma, Two loop neutrino model and dark matter particles with global B-L symmetry, JCAP 06 (2014) 027 [arXiv:1312.3761] [INSPIRE].

[78] H. Okada, Two loop induced Dirac neutrino model and dark matters with global U(1)' symmetry, arXiv:1404.0280 [INSPIRE].

[79] S. Kanemura, T. Matsui and H. Sugiyama, Neutrino mass and dark matter from gauged $U(1)_{B-L}$ breaking, Phys. Rev. D 90 (2014) 013001 [arXiv:1405.1935] [INSPIRE].

[80] M. Aoki and T. Toma, Impact of semi-annihilation of $\mathbb{Z}_{3}$ symmetric dark matter with radiative neutrino masses, JCAP 09 (2014) 016 [arXiv: 1405.5870] [INSPIRE].

[81] H. Okada, T. Toma and K. Yagyu, Inert extension of the Zee-Babu model, Phys. Rev. D 90 (2014) 095005 [arXiv: 1408.0961] [INSPIRE].

[82] D. Aristizabal Sierra, A. Degee, L. Dorame and M. Hirsch, Systematic classification of two-loop realizations of the Weinberg operator, JHEP 03 (2015) 040 [arXiv:1411.7038] [INSPIRE].

[83] H. Okada, Two loop radiative seesaw and X-ray line dark matter with global U(1) symmetry, arXiv: 1503.04557 [INSPIRE]. 
[84] S. Kashiwase, H. Okada, Y. Orikasa and T. Toma, Two loop neutrino model with dark matter and leptogenesis, Int. J. Mod. Phys. A 31 (2016) 1650121 [arXiv:1505.04665] [INSPIRE].

[85] H. Okada and Y. Orikasa, Two-loop neutrino model with exotic leptons, Phys. Rev. D 93 (2016) 013008 [arXiv: 1509.04068] [InSPIRE].

[86] R. Ding, Z.-L. Han, Y. Liao and W.-P. Xie, Radiative neutrino mass with $\mathbb{Z}_{3}$ dark matter: from relic density to LHC signatures, JHEP 05 (2016) 030 [arXiv:1601.06355] [INSPIRE].

[87] T. Nomura and H. Okada, Generalized Zee-Babu model with 750 GeV diphoton resonance, Phys. Lett. B 756 (2016) 295 [arXiv: 1601.07339] [INSPIRE].

[88] T. Nomura, H. Okada and Y. Orikasa, Radiative neutrino mass in alternative left-right model, Eur. Phys. J. C 77 (2017) 103 [arXiv:1602.08302] [INSPIRE].

[89] C. Bonilla, E. Ma, E. Peinado and J.W.F. Valle, Two-loop Dirac neutrino mass and WIMP dark matter, Phys. Lett. B 762 (2016) 214 [arXiv:1607.03931] [INSPIRE].

[90] T. Nomura and H. Okada, An extended colored Zee-Babu model, Phys. Rev. D 94 (2016) 075021 [arXiv: 1607.04952] [INSPIRE].

[91] W.-F. Chang, S.-C. Liou, C.-F. Wong and F. Xu, Charged lepton flavor violating processes and scalar leptoquark decay branching ratios in the colored Zee-Babu model, JHEP 10 (2016) 106 [arXiv:1608.05511] [INSPIRE].

[92] T. Nomura and H. Okada, Two-loop induced Majorana neutrino mass in a radiatively induced quark and lepton mass model, Phys. Rev. D 94 (2016) 093006 [arXiv: 1609.01504] [INSPIRE].

[93] L.M. Krauss, S. Nasri and M. Trodden, A model for neutrino masses and dark matter, Phys. Rev. D 67 (2003) 085002 [hep-ph/0210389] [INSPIRE].

[94] K. Cheung and O. Seto, Phenomenology of TeV right-handed neutrino and the dark matter model, Phys. Rev. D 69 (2004) 113009 [hep-ph/0403003] [InSPIRE].

[95] M. Aoki, S. Kanemura and O. Seto, Neutrino mass, dark matter and baryon asymmetry via TeV-scale physics without fine-tuning, Phys. Rev. Lett. 102 (2009) 051805 [arXiv:0807.0361] [INSPIRE].

[96] M. Gustafsson, J.M. No and M.A. Rivera, Predictive model for radiatively induced neutrino masses and mixings with dark matter, Phys. Rev. Lett. 110 (2013) 211802 [arXiv:1212.4806] [INSPIRE].

[97] J.N. Ng and A. de la Puente, Top quark as a dark portal and neutrino mass generation, Phys. Lett. B 727 (2013) 204 [arXiv:1307.2606] [InSPIRE].

[98] Y. Kajiyama, H. Okada and K. Yagyu, $T_{7}$ flavor model in three loop seesaw and Higgs phenomenology, JHEP 10 (2013) 196 [arXiv: 1307.0480] [INSPIRE].

[99] A. Ahriche, C.-S. Chen, K.L. McDonald and S. Nasri, Three-loop model of neutrino mass with dark matter, Phys. Rev. D 90 (2014) 015024 [arXiv:1404.2696] [INSPIRE].

[100] H. Hatanaka, K. Nishiwaki, H. Okada and Y. Orikasa, A three-loop neutrino model with global U(1) symmetry, Nucl. Phys. B 894 (2015) 268 [arXiv:1412.8664] [INSPIRE].

[101] K. Nishiwaki, H. Okada and Y. Orikasa, Three loop neutrino model with isolated $k^{ \pm \pm}$, Phys. Rev. D 92 (2015) 093013 [arXiv: 1507.02412] [INSPIRE].

[102] H. Okada and K. Yagyu, Three-loop neutrino mass model with doubly charged particles from isodoublets, Phys. Rev. D 93 (2016) 013004 [arXiv: 1508.01046] [INSPIRE]. 
[103] A. Ahriche, K.L. McDonald and S. Nasri, A radiative model for the weak scale and neutrino mass via dark matter, JHEP 02 (2016) 038 [arXiv: 1508.02607] [INSPIRE].

[104] S. Kanemura, K. Nishiwaki, H. Okada, Y. Orikasa, S.C. Park and R. Watanabe, LHC $750 \mathrm{GeV}$ diphoton excess in a radiative seesaw model, PTEP 2016 (2016) 123B04 [arXiv: 1512.09048] [INSPIRE].

[105] H. Okada and K. Yagyu, Renormalizable model for neutrino mass, dark matter, muon $g-2$ and $750 \mathrm{GeV}$ diphoton excess, Phys. Lett. B 756 (2016) 337 [arXiv:1601.05038] [INSPIRE].

[106] P. Ko, T. Nomura, H. Okada and Y. Orikasa, Confronting a new three-loop seesaw model with the $750 \mathrm{GeV}$ diphoton excess, Phys. Rev. D 94 (2016) 013009 [arXiv:1602.07214] [INSPIRE].

[107] T. Nomura, H. Okada and Y. Orikasa, Radiative seesaw model with degenerate Majorana dark matter, Phys. Rev. D 93 (2016) 113008 [arXiv:1603.04631] [INSPIRE].

[108] D. Cherigui, C. Guella, A. Ahriche and S. Nasri, Probing radiative neutrino mass models using trilepton channel at the LHC, Phys. Lett. B 762 (2016) 225 [arXiv:1605.03640] [INSPIRE].

[109] T. Nomura, H. Okada and N. Okada, A colored KNT neutrino model, Phys. Lett. B 762 (2016) 409 [arXiv: 1608.02694] [INSPIRE].

[110] K. Cheung, H. Ishida and H. Okada, Accommodation of the Dirac phase in the Krauss-Nasri-Trodden model, arXiv: 1609.06231 [INSPIRE].

[111] K. Cheung, T. Nomura and H. Okada, Three-loop neutrino mass model with a colored triplet scalar, Phys. Rev. D 95 (2017) 015026 [arXiv: 1610.04986] [INSPIRE].

[112] S.M. Boucenna, S. Morisi and J.W.F. Valle, The low-scale approach to neutrino masses, Adv. High Energy Phys. 2014 (2014) 831598 [arXiv: 1404.3751] [INSPIRE].

[113] M.-C. Chen and J. Huang, TeV scale models of neutrino masses and their phenomenology, Mod. Phys. Lett. A 26 (2011) 1147 [arXiv:1105.3188] [INSPIRE].

[114] E. Ma, Naturally small seesaw neutrino mass with no new physics beyond the TeV scale, Phys. Rev. Lett. 86 (2001) 2502 [hep-ph/0011121] [INSPIRE].

[115] E. Ma and D.P. Roy, Heavy triplet leptons and new gauge boson, Nucl. Phys. B 644 (2002) 290 [hep-ph/0206150] [INSPIRE].

[116] F. Wang, W. Wang and J.M. Yang, Split two-Higgs-doublet model and neutrino condensation, Europhys. Lett. 76 (2006) 388 [hep-ph/0601018] [INSPIRE].

[117] S. Gabriel and S. Nandi, A new two Higgs doublet model, Phys. Lett. B 655 (2007) 141 [hep-ph/0610253] [INSPIRE].

[118] S.M. Davidson and H.E. Logan, Dirac neutrinos from a second Higgs doublet, Phys. Rev. D 80 (2009) 095008 [arXiv:0906.3335] [InSPIRE].

[119] P. Bandyopadhyay, S. Choubey and M. Mitra, Two Higgs doublet type III seesaw with $\mu-\tau$ symmetry at LHC, JHEP 10 (2009) 012 [arXiv:0906.5330] [INSPIRE].

[120] G. Marshall, M. McCaskey and M. Sher, A supersymmetric model with Dirac neutrino masses, Phys. Rev. D 81 (2010) 053006 [arXiv:0912.1599] [InSPIRE].

[121] N. Haba and K. Tsumura, $\nu$-two Higgs doublet model and its collider phenomenology, JHEP 06 (2011) 068 [arXiv:1105.1409] [INSPIRE].

[122] N. Haba, K. Kaneta and Y. Shimizu, Phenomenology of supersymmetry SU(5) GUT with neutrinophilic Higgs boson, Phys. Rev. D 86 (2012) 015019 [arXiv:1204.4254] [INSPIRE]. 
[123] U. Maitra, B. Mukhopadhyaya, S. Nandi, S.K. Rai and A. Shivaji, Searching for an elusive charged Higgs boson at the Large Hadron Collider, Phys. Rev. D 89 (2014) 055024 [arXiv:1401.1775] [INSPIRE].

[124] S. Chakdar, K. Ghosh and S. Nandi, A predictive model of Dirac neutrinos, Phys. Lett. B 734 (2014) 64 [arXiv: 1403.1544] [INSPIRE].

[125] O. Seto, Large invisible decay of a Higgs boson to neutrinos, Phys. Rev. D 92 (2015) 073005 [arXiv: 1507.06779] [INSPIRE].

[126] F.-X. Josse-Michaux and E. Molinaro, A common framework for dark matter, leptogenesis and neutrino masses, Phys. Rev. D 84 (2011) 125021 [arXiv:1108.0482] [INSPIRE].

[127] P. Mitropoulos, Right-handed sneutrinos as asymmetric DM and neutrino masses from neutrinophilic Higgs bosons, JCAP 11 (2013) 008 [arXiv:1307.2823] [INSPIRE].

[128] K.-Y. Choi, O. Seto and C.S. Shin, Phenomenology in supersymmetric neutrinophilic Higgs model with sneutrino dark matter, JHEP 09 (2014) 068 [arXiv: 1406.0228] [INSPIRE].

[129] A. Adulpravitchai and M.A. Schmidt, Sterile neutrino dark matter production in the neutrino-phillic two Higgs doublet model, JHEP 12 (2015) 023 [arXiv: 1507.05694] [INSPIRE].

[130] S. Baek and T. Nomura, Dark matter physics in neutrino specific two Higgs doublet model, JHEP 03 (2017) 059 [arXiv: 1611.09145] [INSPIRE].

[131] N. Haba and O. Seto, Low scale thermal leptogenesis in neutrinophilic Higgs doublet models, Prog. Theor. Phys. 125 (2011) 1155 [arXiv:1102.2889] [InSPIRE].

[132] N. Haba and O. Seto, Thermal leptogenesis in a supersymmetric neutrinophilic Higgs model, Phys. Rev. D 84 (2011) 103524 [arXiv:1106.5354] [INSPIRE].

[133] W. Chao and M.J. Ramsey-Musolf, Hidden from view: Neutrino masses, dark matter and TeV-scale leptogenesis in a neutrinophilic two-Higgs-doublet model, Phys. Rev. D 89 (2014) 033007 [arXiv: 1212.5709] [INSPIRE].

[134] N. Haba, O. Seto and Y. Yamaguchi, Neutrino mass from neutrinophilic Higgs and leptogenesis, arXiv:1401.6646 [INSPIRE].

[135] J.D. Clarke, R. Foot and R.R. Volkas, Natural leptogenesis and neutrino masses with two Higgs doublets, Phys. Rev. D 92 (2015) 033006 [arXiv: 1505.05744] [INSPIRE].

[136] N. Haba and M. Hirotsu, TeV-scale seesaw from a multi-Higgs model, Eur. Phys. J. C 69 (2010) 481 [arXiv: 1005.1372] [inSPIRE].

[137] A. Datta, M. Guchait and A. Pilaftsis, Probing lepton number violation via Majorana neutrinos at hadron supercolliders, Phys. Rev. D 50 (1994) 3195 [hep-ph/9311257] [INSPIRE].

[138] F.M.L. Almeida, Jr., Y. do Amaral Coutinho, J.A. Martins Simoes and M.A.B. do Vale, On a signature for heavy Majorana neutrinos in hadronic collisions, Phys. Rev. D 62 (2000) 075004 [hep-ph/0002024] [INSPIRE].

[139] O. Panella, M. Cannoni, C. Carimalo and Y.N. Srivastava, Signals of heavy Majorana neutrinos at hadron colliders, Phys. Rev. D 65 (2002) 035005 [hep-ph/0107308] [InSPIRE].

[140] T. Han and B. Zhang, Signatures for Majorana neutrinos at hadron colliders, Phys. Rev. Lett. 97 (2006) 171804 [hep-ph/0604064] [INSPIRE].

[141] F. del Aguila and J.A. Aguilar-Saavedra, Distinguishing seesaw models at LHC with multi-lepton signals, Nucl. Phys. B $\mathbf{8 1 3}$ (2009) 22 [arXiv:0808.2468] [INSPIRE]. 
[142] A. Atre, T. Han, S. Pascoli and B. Zhang, The search for heavy Majorana neutrinos, JHEP 05 (2009) 030 [arXiv:0901.3589] [InSPIRE].

[143] A. Das and N. Okada, Inverse seesaw neutrino signatures at the LHC and ILC, Phys. Rev. D 88 (2013) 113001 [arXiv:1207.3734] [INSPIRE].

[144] A. Das, P.S. Bhupal Dev and N. Okada, Direct bounds on electroweak scale pseudo-Dirac neutrinos from $\sqrt{s}=8 \mathrm{TeV}$ LHC data, Phys. Lett. B 735 (2014) 364 [arXiv:1405.0177] [INSPIRE].

[145] P.S.B. Dev, A. Pilaftsis and U.-k. Yang, New production mechanism for heavy neutrinos at the LHC, Phys. Rev. Lett. 112 (2014) 081801 [arXiv: 1308. 2209] [INSPIRE].

[146] D. Alva, T. Han and R. Ruiz, Heavy Majorana neutrinos from $W \gamma$ fusion at hadron colliders, JHEP 02 (2015) 072 [arXiv: 1411.7305] [INSPIRE].

[147] S. Banerjee, P.S.B. Dev, A. Ibarra, T. Mandal and M. Mitra, Prospects of heavy neutrino searches at future lepton colliders, Phys. Rev. D 92 (2015) 075002 [arXiv:1503.05491] [INSPIRE].

[148] J.N. Ng, A. de la Puente and B. W.-P. Pan, Search for heavy right-handed neutrinos at the LHC and beyond in the same-sign same-flavor leptons final state, JHEP 12 (2015) 172 [arXiv: 1505.01934] [INSPIRE].

[149] A. Das and N. Okada, Improved bounds on the heavy neutrino productions at the LHC, Phys. Rev. D 93 (2016) 033003 [arXiv: 1510.04790] [InSPIRE].

[150] R. Leonardi, L. Alunni, F. Romeo, L. Fanò and O. Panella, Hunting for heavy composite Majorana neutrinos at the LHC, Eur. Phys. J. C 76 (2016) 593 [arXiv:1510.07988] [INSPIRE].

[151] A. Das, P. Konar and S. Majhi, Production of heavy neutrino in next-to-leading order QCD at the LHC and beyond, JHEP 06 (2016) 019 [arXiv: 1604.00608] [INSPIRE].

[152] F.F. Deppisch, P.S. Bhupal Dev and A. Pilaftsis, Neutrinos and collider physics, New J. Phys. 17 (2015) 075019 [arXiv: 1502.06541] [InSPIRE].

[153] J. Chakrabortty, J. Gluza, R. Sevillano and R. Szafron, Left-right symmetry at LHC and precise 1-loop low energy data, JHEP 07 (2012) 038 [arXiv:1204.0736] [INSPIRE].

[154] J. Gluza, T. Jelinski and R. Szafron, Lepton number violation and 'Diracness' of massive neutrinos composed of Majorana states, Phys. Rev. D 93 (2016) 113017 [arXiv: 1604.01388] [INSPIRE].

[155] S. Antusch, E. Cazzato and O. Fischer, Sterile neutrino searches at future $e^{-} e^{+}, p p$ and $e^{-} p$ colliders, arXiv:1612.02728 [inSPIRE].

[156] Z.-z. Xing, Naturalness and testability of TeV seesaw mechanisms, Prog. Theor. Phys. Suppl. 180 (2009) 112 [arXiv: 0905. 3903] [INSPIRE].

[157] X.-G. He, S. Oh, J. Tandean and C.-C. Wen, Large mixing of light and heavy neutrinos in seesaw models and the LHC, Phys. Rev. D 80 (2009) 073012 [arXiv: 0907.1607] [INSPIRE].

[158] W.-Y. Keung and G. Senjanović, Majorana neutrinos and the production of the right-handed charged gauge boson, Phys. Rev. Lett. 50 (1983) 1427 [INSPIRE].

[159] K. Huitu, S. Khalil, H. Okada and S.K. Rai, Signatures for right-handed neutrinos at the Large Hadron Collider, Phys. Rev. Lett. 101 (2008) 181802 [arXiv:0803.2799] [INSPIRE].

[160] L. Basso, A. Belyaev, S. Moretti and C.H. Shepherd-Themistocleous, Phenomenology of the minimal B-L extension of the standard model: $Z^{\prime}$ and neutrinos, Phys. Rev. D 80 (2009) 055030 [arXiv: 0812.4313] [INSPIRE]. 
[161] D.G. Cerdeno and O. Seto, Right-handed sneutrino dark matter in the NMSSM, JCAP 08 (2009) 032 [arXiv: 0903.4677] [inSPIRE].

[162] L. Basso, Phenomenology of the minimal B-L extension of the standard model at the LHC, arXiv:1106.4462 [INSPIRE].

[163] C.-Y. Chen and P.S.B. Dev, Multi-lepton collider signatures of heavy Dirac and Majorana neutrinos, Phys. Rev. D 85 (2012) 093018 [arXiv:1112.6419] [INSPIRE].

[164] C.-Y. Chen, P.S.B. Dev and R.N. Mohapatra, Probing heavy-light neutrino mixing in left-right seesaw models at the LHC, Phys. Rev. D 88 (2013) 033014 [arXiv:1306.2342] [INSPIRE].

[165] S.P. Das, F.F. Deppisch, O. Kittel and J.W.F. Valle, Heavy neutrinos and lepton flavour violation in left-right symmetric models at the LHC, Phys. Rev. D 86 (2012) 055006 [arXiv:1206.0256] [inSPIRE].

[166] T. Han, I. Lewis, R. Ruiz and Z.-g. Si, Lepton number violation and $W^{\prime}$ chiral couplings at the LHC, Phys. Rev. D 87 (2013) 035011 [arXiv:1211.6447] [InSPIRE].

[167] J. Gluza and T. Jeliński, Heavy neutrinos and the $p p \rightarrow l l j j$ CMS data, Phys. Lett. B 748 (2015) 125 [arXiv: 1504.05568] [INSPIRE].

[168] A.M. Gago, P. Hernández, J. Jones-Pérez, M. Losada and A. Moreno Briceño, Probing the type I seesaw mechanism with displaced vertices at the LHC, Eur. Phys. J. C 75 (2015) 470 [arXiv: 1505.05880] [INSPIRE].

[169] Z. Kang, P. Ko and J. Li, New avenues to heavy right-handed neutrinos with pair production at hadronic colliders, Phys. Rev. D 93 (2016) 075037 [arXiv:1512.08373] [INSPIRE].

[170] M. Lindner, F.S. Queiroz, W. Rodejohann and C.E. Yaguna, Left-right symmetry and lepton number violation at the large hadron electron collider, JHEP 06 (2016) 140 [arXiv: 1604.08596] [INSPIRE].

[171] M. Mitra, R. Ruiz, D.J. Scott and M. Spannowsky, Neutrino jets from high-mass $W_{R}$ gauge bosons in TeV-scale left-right symmetric models, Phys. Rev. D 94 (2016) 095016 [arXiv: 1607.03504] [INSPIRE].

[172] E. Accomando, L. Delle Rose, S. Moretti, E. Olaiya and C. Shepherd-Themistocleous, Novel SM-like Higgs decay into displaced heavy neutrino pairs in $\mathrm{U}(1)^{\prime}$ models, arXiv: 1612.05977 [INSPIRE].

[173] W. Wang and Z.-L. Han, Global U(1) $)_{L}$ breaking in neutrinophilic 2HDM: from LHC signatures to X-ray line, Phys. Rev. D 94 (2016) 053015 [arXiv: 1605.00239] [INSPIRE].

[174] G.C. Branco, P.M. Ferreira, L. Lavoura, M.N. Rebelo, M. Sher and J.P. Silva, Theory and phenomenology of two-Higgs-doublet models, Phys. Rept. 516 (2012) 1 [arXiv:1106.0034] [INSPIRE].

[175] J.F. Gunion and H.E. Haber, The CP conserving two Higgs doublet model: the approach to the decoupling limit, Phys. Rev. D 67 (2003) 075019 [hep-ph/0207010] [INSPIRE].

[176] T. Morozumi, H. Takata and K. Tamai, Quantum correction to tiny vacuum expectation value in two Higgs doublet model for Dirac neutrino mass, Phys. Rev. D 85 (2012) 055002 [arXiv:1107.1026] [INSPIRE].

[177] N. Haba and T. Horita, Vacuum stability in neutrinophilic Higgs doublet model, Phys. Lett. B 705 (2011) 98 [arXiv:1107.3203] [INSPIRE].

[178] ATLAS collaboration, Observation of a new particle in the search for the standard model Higgs boson with the ATLAS detector at the LHC, Phys. Lett. B 716 (2012) 1 [arXiv: 1207.7214] [INSPIRE]. 
[179] CMS collaboration, Observation of a new boson at a mass of $125 \mathrm{GeV}$ with the CMS experiment at the LHC, Phys. Lett. B 716 (2012) 30 [arXiv:1207.7235] [INSPIRE].

[180] ATLAS, CMS collaboration, Combined measurement of the Higgs boson mass in pp collisions at $\sqrt{s}=7$ and $8 \mathrm{TeV}$ with the ATLAS and CMS experiments, Phys. Rev. Lett. 114 (2015) 191803 [arXiv: 1503.07589] [INSPIRE].

[181] P.A.N. Machado, Y.F. Perez, O. Sumensari, Z. Tabrizi and R.Z. Funchal, On the viability of minimal neutrinophilic two-Higgs-doublet models, JHEP 12 (2015) 160 [arXiv:1507.07550] [INSPIRE].

[182] D.V. Forero, M. Tortola and J.W.F. Valle, Neutrino oscillations refitted, Phys. Rev. D 90 (2014) 093006 [arXiv: 1405.7540] [InSPIRE].

[183] J.A. Casas and A. Ibarra, Oscillating neutrinos and muon $\rightarrow e, \gamma$, Nucl. Phys. B 618 (2001) 171 [hep-ph/0103065] [INSPIRE].

[184] A. Ibarra and G.G. Ross, Neutrino phenomenology: the case of two right-handed neutrinos, Phys. Lett. B 591 (2004) 285 [hep-ph/0312138] [INSPIRE].

[185] P. Fileviez Perez, T. Han and T. Li, Testability of type I seesaw at the CERN LHC: revealing the existence of the B-L symmetry, Phys. Rev. D 80 (2009) 073015 [arXiv:0907.4186] [INSPIRE].

[186] ATLAS, CMS collaboration, Measurements of the Higgs boson production and decay rates and constraints on its couplings from a combined ATLAS and CMS analysis of the LHC pp collision data at $\sqrt{s}=7$ and $8 \mathrm{TeV}$, JHEP 08 (2016) 045 [arXiv: 1606. 02266] [INSPIRE].

[187] J. Lesgourgues and S. Pastor, Neutrino mass from cosmology, Adv. High Energy Phys. 2012 (2012) 608515 [arXiv:1212.6154] [INSPIRE].

[188] Planck collaboration, P.A.R. Ade et al., Planck 2013 results. XVI. Cosmological parameters, Astron. Astrophys. 571 (2014) A16 [arXiv:1303.5076] [INSPIRE].

[189] S.M. Bilenky and C. Giunti, Neutrinoless double-beta decay: a brief review, Mod. Phys. Lett. A 27 (2012) 1230015 [arXiv: 1203.5250] [INSPIRE].

[190] S. Dell'Oro, S. Marcocci, M. Viel and F. Vissani, Neutrinoless double beta decay: 2015 review, Adv. High Energy Phys. 2016 (2016) 2162659 [arXiv: 1601.07512] [INSPIRE].

[191] EXO-200 collaboration, M. Auger et al., Search for neutrinoless double-beta decay in ${ }^{136}$ Xe with EXO-200, Phys. Rev. Lett. 109 (2012) 032505 [arXiv: 1205.5608] [INSPIRE].

[192] EXO-200 collaboration, J.B. Albert et al., Search for Majorana neutrinos with the first two years of EXO-200 data, Nature $\mathbf{5 1 0}$ (2014) 229 [arXiv:1402.6956] [INSPIRE].

[193] KamLAND-Zen collaboration, A. Gando et al., Limit on neutrinoless $\beta \beta$ decay of ${ }^{136}$ Xe from the first phase of KamLAND-Zen and comparison with the positive claim in ${ }^{76} \mathrm{Ge}$, Phys. Rev. Lett. 110 (2013) 062502 [arXiv:1211.3863] [InSPIRE].

[194] KamLAND-Zen collaboration, A. Gando et al., Search for Majorana neutrinos near the inverted mass hierarchy region with KamLAND-Zen, Phys. Rev. Lett. 117 (2016) 082503 [arXiv: 1605. 02889] [INSPIRE].

[195] E. Ma and M. Raidal, Neutrino mass, muon anomalous magnetic moment and lepton flavor nonconservation, Phys. Rev. Lett. 87 (2001) 011802 [Erratum ibid. 87 (2001) 159901] [hep-ph/0102255] [INSPIRE].

[196] E. Bertuzzo, Y.F. Perez G., O. Sumensari and R. Zukanovich Funchal, Limits on neutrinophilic two-Higgs-doublet models from flavor physics, JHEP 01 (2016) 018 [arXiv: 1510.04284] [INSPIRE]. 
[197] MEG collaboration, J. Adam et al., New constraint on the existence of the $\mu^{+} \rightarrow e^{+} \gamma$ decay, Phys. Rev. Lett. 110 (2013) 201801 [arXiv:1303.0754] [INSPIRE].

[198] MEG collaboration, A.M. Baldini et al., Search for the lepton flavour violating decay $\mu^{+} \rightarrow \mathrm{e}^{+} \gamma$ with the full dataset of the MEG experiment, Eur. Phys. J. C 76 (2016) 434 [arXiv: 1605. 05081] [INSPIRE].

[199] R. Ding, Z.-L. Han, Y. Liao, H.-J. Liu and J.-Y. Liu, Phenomenology in the minimal cascade seesaw mechanism for neutrino masses, Phys. Rev. D 89 (2014) 115024 [arXiv: 1403.2040] [INSPIRE].

[200] T. Toma and A. Vicente, Lepton flavor violation in the scotogenic model, JHEP 01 (2014) 160 [arXiv:1312.2840] [INSPIRE].

[201] T. Fukuyama, H. Sugiyama and K. Tsumura, Constraints from muon $g-2$ and LFV processes in the Higgs triplet model, JHEP 03 (2010) 044 [arXiv:0909.4943] [INSPIRE].

[202] Z.-L. Han, R. Ding and Y. Liao, LHC phenomenology of type II seesaw: nondegenerate case, Phys. Rev. D 91 (2015) 093006 [arXiv:1502.05242] [INSPIRE].

[203] Z.-L. Han, R. Ding and Y. Liao, LHC phenomenology of the type-II seesaw mechanism: observability of neutral scalars in the nondegenerate case, Phys. Rev. D 92 (2015) 033014 [arXiv: 1506. 08996] [INSPIRE].

[204] S.M. Davidson and H.E. Logan, LHC phenomenology of a two-Higgs-doublet neutrino mass model, Phys. Rev. D 82 (2010) 115031 [arXiv: 1009.4413] [InSPIRE].

[205] ATLAS collaboration, Search for direct production of charginos, neutralinos and sleptons in final states with two leptons and missing transverse momentum in pp collisions at $\sqrt{s}=8 \mathrm{TeV}$ with the ATLAS detector, JHEP 05 (2014) 071 [arXiv:1403.5294] [INSPIRE].

[206] CMS collaboration, Searches for electroweak production of charginos, neutralinos and sleptons decaying to leptons and $W, Z$ and Higgs bosons in pp collisions at 8 TeV, Eur. Phys. J. C 74 (2014) 3036 [arXiv:1405.7570] [InSPIRE].

[207] LEP, DELPHI, OPAL, ALEPH, L3 collaboration, G. Abbiendi et al., Search for charged Higgs bosons: combined results using LEP data, Eur. Phys. J. C 73 (2013) 2463 [arXiv: 1301.6065] [INSPIRE].

[208] DELPHI collaboration, P. Abreu et al., Search for neutral heavy leptons produced in $Z$ decays, Z. Phys. C 74 (1997) 57 [Erratum ibid. C 75 (1997) 580] [InSPIRE].

[209] L3 collaboration, P. Achard et al., Search for heavy isosinglet neutrino in $e^{+} e^{-}$annihilation at LEP, Phys. Lett. B 517 (2001) 67 [hep-ex/0107014] [INSPIRE].

[210] CMS collaboration, Search for heavy Majorana neutrinos in $\mu^{ \pm} \mu^{ \pm}+$jets and $e^{ \pm} e^{ \pm}+$jets events in pp collisions at $\sqrt{s}=7$ TeV, Phys. Lett. B 717 (2012) 109 [arXiv:1207.6079] [INSPIRE].

[211] ATLAS collaboration, Search for heavy Majorana neutrinos with the ATLAS detector in pp collisions at $\sqrt{s}=8 \mathrm{TeV}$, JHEP 07 (2015) 162 [arXiv:1506.06020] [INSPIRE].

[212] CMS collaboration, Search for heavy Majorana neutrinos in $e^{ \pm} e^{ \pm}+$jets and $e^{ \pm} \mu^{ \pm}+$jets events in proton-proton collisions at $\sqrt{s}=8 \mathrm{TeV}$, JHEP 04 (2016) 169 [arXiv: 1603. 02248] [INSPIRE].

[213] N.D. Christensen and C. Duhr, FeynRules - Feynman rules made easy, Comput. Phys. Commun. 180 (2009) 1614 [arXiv:0806.4194] [INSPIRE].

[214] N.D. Christensen et al., A comprehensive approach to new physics simulations, Eur. Phys. J. C 71 (2011) 1541 [arXiv:0906.2474] [INSPIRE]. 
[215] A. Alloul, N.D. Christensen, C. Degrande, C. Duhr and B. Fuks, FeynRules 2.0 - A complete toolbox for tree-level phenomenology, Comput. Phys. Commun. 185 (2014) 2250 [arXiv:1310.1921] [INSPIRE].

[216] C. Degrande, C. Duhr, B. Fuks, D. Grellscheid, O. Mattelaer and T. Reiter, UFO - The Universal FeynRules Output, Comput. Phys. Commun. 183 (2012) 1201 [arXiv:1108.2040] [INSPIRE].

[217] J. Alwall, M. Herquet, F. Maltoni, O. Mattelaer and T. Stelzer, MadGraph 5: going beyond, JHEP 06 (2011) 128 [arXiv:1106.0522] [INSPIRE].

[218] J. Alwall et al., The automated computation of tree-level and next-to-leading order differential cross sections and their matching to parton shower simulations, JHEP $\mathbf{0 7}$ (2014) 079 [arXiv: 1405.0301] [InSPIRE].

[219] R.D. Ball et al., Parton distributions with LHC data, Nucl. Phys. B 867 (2013) 244 [arXiv: 1207.1303] [INSPIRE].

[220] NNPDF collaboration, R.D. Ball et al., Parton distributions for the LHC Run II, JHEP 04 (2015) 040 [arXiv: 1410.8849] [INSPIRE].

[221] T. Sjöstrand, S. Mrenna and P.Z. Skands, PYTHIA 6.4 physics and manual, JHEP 05 (2006) 026 [hep-ph/0603175] [INSPIRE].

[222] S. Ovyn, X. Rouby and V. Lemaitre, DELPHES, a framework for fast simulation of a generic collider experiment, arXiv:0903.2225 [INSPIRE].

[223] DELPHES 3 collaboration, J. de Favereau et al., DELPHES 3, a modular framework for fast simulation of a generic collider experiment, JHEP 02 (2014) 057 [arXiv:1307.6346] [INSPIRE].

[224] E. Conte, B. Fuks and G. Serret, MadAnalysis 5, a user-friendly framework for collider phenomenology, Comput. Phys. Commun. 184 (2013) 222 [arXiv:1206.1599] [INSPIRE].

[225] E. Conte, B. Dumont, B. Fuks and C. Wymant, Designing and recasting LHC analyses with MadAnalysis 5, Eur. Phys. J. C 74 (2014) 3103 [arXiv:1405.3982] [INSPIRE].

[226] B. Dumont et al., Toward a public analysis database for LHC new physics searches using MADANALYSIS 5, Eur. Phys. J. C 75 (2015) 56 [arXiv:1407.3278] [InSPIRE].

[227] M. Drees, H. Dreiner, D. Schmeier, J. Tattersall and J.S. Kim, CheckMATE: confronting your favourite new physics model with LHC data, Comput. Phys. Commun. 187 (2015) 227 [arXiv: 1312.2591] [INSPIRE].

[228] D. Dercks, N. Desai, J.S. Kim, K. Rolbiecki, J. Tattersall and T. Weber, CheckMATE 2: from the model to the limit, arXiv:1611.09856 [INSPIRE].

[229] S. Hoeche et al., Matching parton showers and matrix elements, hep-ph/0602031 [INSPIRE].

[230] CMS collaboration, Identification of b-quark jets with the CMS experiment, 2013 JINST 8 P04013 [arXiv: 1211.4462] [INSPIRE].

[231] ATLAS collaboration, Performance of b-jet identification in the ATLAS experiment, 2016 JINST 11 P04008 [arXiv:1512.01094] [INSPIRE]. 